\title{
Distance problems for dissipative Hamiltonian systems and related matrix polynomials
}

\author{
C. Mehl ${ }^{\ddagger *} \quad$ V. Mehrmann ${ }^{\ddagger *} \quad$ M. Wojtylak ${ }^{\dagger \S}$ \\ January 27, 2020 \\ Dedicated to Paul Van Dooren on the occasion of his 70th birthday
}

\begin{abstract}
We study the characterization of several distance problems for linear differentialalgebraic systems with dissipative Hamiltonian structure. Since all models are only approximations of reality and data are always inaccurate, it is an important question whether a given model is close to a 'bad' model that could be considered as ill-posed or singular. This is usually done by computing a distance to the nearest model with such properties. We will discuss the distance to singularity and the distance to the nearest high index problem for dissipative Hamiltonian systems. While for general unstructured differentialalgebraic systems the characterization of these distances are partially open problems, we will show that for dissipative Hamiltonian systems and related matrix polynomials there exist explicit characterizations that can be implemented numerically.
\end{abstract}

Keywords. distance to singularity, distance to high index problem, distance to instability, dissipative Hamiltonian system, differential-algebraic system, matrix pencil, Kronecker canonical form,

AMS subject classification 2014. 15A18, 15A21, 15A22

\section{Introduction}

We study several distance problems for linear systems of differential-algebraic equations (DAEs) of the form

$$
E \dot{x}=(J-R) Q x,
$$

with constant coefficient matrices $E, Q, J, R \in \mathbb{R}^{n, n}$ and a differentiable state function $x$ : $\mathbb{R} \rightarrow \mathbb{R}^{n}$, see also [3, 16, 22, 30, 31, 32, 33, 34, for definitions and a detailed analysis of such systems in different generality and their relation to the more general port-Hamiltonian

\footnotetext{
${ }^{\ddagger}$ Institut für Mathematik, MA 4-5, TU Berlin, Str. des 17 . Juni 136, D-10623 Berlin, FRG. \{mehl, mehrmann\}@math.tu-berlin.de.

${ }^{\dagger}$ Instytut Matematyki, Wydział Matematyki i Informatyki, Uniwersytet Jagielloński, Kraków, ul. Łojasiewicza 6, 30-348 Kraków, Poland michal.wojtylak@uj.edu.pl.

${ }^{\S}$ Supported by the Alexander von Humboldt Foundation.

${ }^{*}$ Partially supported by Deutsche Forschungsgemeinschaft through the Excellence Cluster MATH ${ }^{+}$in Berlin and Priority Program 1984 'Hybride und multimodale Energiesysteme: Systemtheoretische Methoden fr die Transformation und den Betrieb komplexer Netze'.
} 
systems. A system of the above form is called linear time-invariant dissipative Hamiltonian $(d H)$ differential-algebraic equation (dHDAE) system if

$$
E^{\top} Q \geq 0, \quad J=-J^{\top}, \quad R=R^{\top} \geq 0,
$$

where $A^{\top}$ denotes the transpose of a matrix $A$ and for a symmetric matrix $A=A^{\top}$ by $A>0(A \geq 0)$ we denote that $A$ is positive definite (positive semidefinite). Such dHDAE systems generalize linear time-invariant ordinary dissipative Hamiltonian systems (the case where $E=I$ ) is the identity) and linear Hamiltonian systems, the case that $E=I$ and $R=0$. The associated quadratic Hamiltonian is given by $\mathcal{H}(x)=\frac{1}{2} x^{\top} E^{\top} Q x$ and satisfies the dissipation inequality $\mathcal{H}\left(x\left(t_{1}\right)\right)-\mathcal{H}\left(x\left(t_{0}\right)\right) \leq 0$ for $t_{1} \geq t_{0}$. In many applications the matrix $Q$ can be chosen to be the identity matrix, i.e., $Q=I$, see [3, 16, 31, 32, 35], and this is the case that we study in this paper. In Section 6.3 we provide an analysis how the general case (11) can be transformed to this situation.

The system properties of (10) (with $Q=I$ ) can be analyzed by investigating the corresponding dH matrix pencil

$$
L(\lambda):=\lambda E-(J-R) .
$$

In our analysis we focus on systems with real coefficients. Some of our results can also easily be extended to the case of complex coefficients, but in some occasions we make explicit or implicit use of the fact that skew-symmetric matrices have a zero diagonal which is not true for skew-Hermitian matrices.

In many practical cases, see, e.g., [3, 20], the underlying system is of second order form $M \ddot{x}-(G-D) \dot{x}+K x=0$ with the underlying quadratic matrix polynomial

$$
P(\lambda):=\lambda^{2} M-\lambda(G-D)+K
$$

where $M, G, D, K \in \mathbb{R}^{n, n}$ satisfy $M=M^{\top}, D=D^{\top}, K=K^{\top} \geq 0$ and $G=-G^{\top}$. As we will see below, this can be viewed as a generalization of the $\mathrm{dH}$ structure to second order systems. The second order case can be easily rewritten in first order $\mathrm{dH}$ form but we will treat the problem directly in second order, and we will also discuss appropriate higher degree matrix polynomials $P(\lambda)$ with an analogous structure.

Linear time invariant systems with the described structure are very common in all areas of science and engineering [3, 31, 35] and typically arise via linearization arround a stationary solution. However, since all mathematical models of physical systems are usually only approximations of reality and data are typically inaccurate, it is an important question whether a given model is close to a model with 'bad properties' such as an ill-posed model without or with non-unique solution. To answer such questions for $\mathrm{dH}$ and port-Hamiltonian systems has been an important research topic in recent years, see, e.g., [1, 2, 16, 17, 18, 27, 28].

To classify whether a model is close to a 'bad model', one usually computes the distance to the nearest model with the 'bad' property. In this paper we will discuss the distance to the set of singular matrix polynomials, i.e., those with a determinant that is identically zero, and the distance to the nearest high-index problem, i.e., a problem with Jordan blocks associated to the eigenvalue $\infty$ of size bigger than one.

While for general unstructured DAE systems the characterization of these two distances is very difficult and partially open [4, 5, 7, 21, 29], the picture changes if one considers structured distances, i.e., distances within the set of linear constant coefficient dHDAE systems. In this paper, we will make use of previous results from [16, 30] to derive explicit characterizations for computing these distances in terms of null-spaces of several matrices. 
We use the following notation. By $\|X\|_{F}$ we denote the Frobenius norm of a (possibly rectangular) matrix $X$, we extend this norm to matrix polynomials $P(\lambda)=\sum_{j=0}^{k} \lambda^{j} X_{j}$ by setting $\|P(\lambda)\|_{F}=\left\|\left[X_{0}, \ldots, X_{k}\right]\right\|_{F}$. By $\lambda_{\min }(X)$ we denote the smallest eigenvalue of a positive semidefinite matrix $X$.

The paper is organized as follows. In Section 2 we recall a few basic results about linear time-invariant $\mathrm{dH}$ systems. In Section 3 we present the different distances and state the main results for first order systems. Instead of immediately presenting the corresponding proofs, we first consider related distance problems for a more general polynomial structure in Section 4 . These distance characterizations are then specialized in Section 5 to prove the main results for the first order case. In Section [6 we consider corresponding distances for analogous quadratic matrix polynomials and also show how different representations of the first order case can be related.

\section{Preliminaries}

We will make use of the Kronecker canonical form of a matrix pencil [15. Let us denote by $\mathcal{J}_{n}\left(\lambda_{0}\right)$ the standard upper triangular Jordan block of size $n \times n$ associated with the eigenvalue $\lambda_{0}$ and let $\mathcal{L}_{n}$ denote the standard right Kronecker block of size $n \times(n+1)$, i.e.,

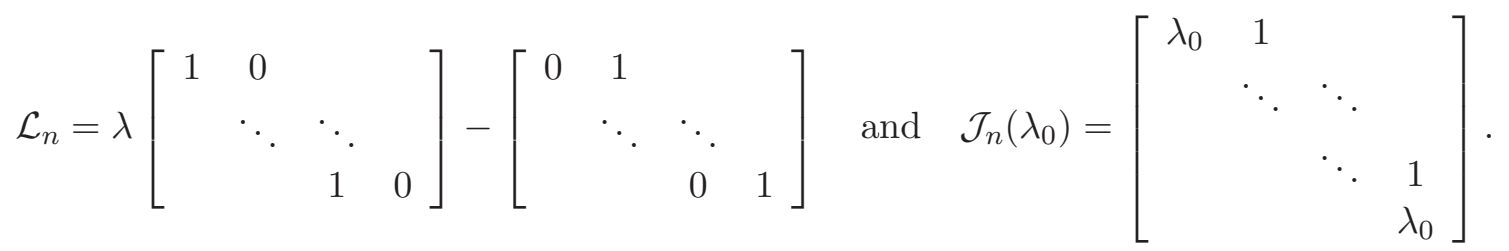

Theorem 1 (Kronecker canonical form) Let $E, A \in \mathbb{C}^{n, m}$. Then there exist nonsingular matrices $S \in \mathbb{C}^{n, n}$ and $T \in \mathbb{C}^{m, m}$ such that

$$
S(\lambda E-A) T=\operatorname{diag}\left(\mathcal{L}_{\epsilon_{1}}, \ldots, \mathcal{L}_{\epsilon_{p}}, \mathcal{L}_{\eta_{1}}^{\top}, \ldots, \mathcal{L}_{\eta_{q}}^{\top}, \mathcal{J}_{\rho_{1}}^{\lambda_{1}}, \ldots, \mathcal{J}_{\rho_{r}}^{\lambda_{r}}, \mathcal{N}_{\sigma_{1}}, \ldots, \mathcal{N}_{\sigma_{s}}\right),
$$

where $p, q, r, s, \epsilon_{1}, \ldots, \epsilon_{p}, \eta_{1}, \ldots, \eta_{q}, \rho_{1}, \ldots, \rho_{r}, \sigma_{1}, \ldots, \sigma_{s} \in \mathbb{N}$ and $\lambda_{1}, \ldots, \lambda_{r} \in \mathbb{C}$, as well as $\mathcal{J}_{\rho_{i}}^{\lambda_{i}}=I_{\rho_{i}}-\mathcal{J}_{\rho_{i}}\left(\lambda_{i}\right)$ for $i=1, \ldots, r$ and $\mathcal{N}_{\sigma_{j}}=\mathcal{J}_{\sigma_{j}}(0)-I_{\sigma_{j}}$ for $j=1, \ldots, s$. This form $i s$ unique up to permutation of the blocks.

For real matrices (the case we discuss), a real version of the Kronecker canonical form is obtained under real transformation matrices $S, T$. In this case the blocks $\mathcal{J}_{\rho_{j}}^{\lambda_{j}}$ with $\lambda_{j} \in \mathbb{C} \backslash \mathbb{R}$ have to be replaced with corresponding blocks in real Jordan canonical form associated to the corresponding pair of conjugate complex eigenvalues, but the other blocks have the same structure as in the complex case. An eigenvalue is called semisimple if the largest associated Jordan block has size one.

The sizes $\eta_{j}$ and $\epsilon_{i}$ of the rectangular blocks are called the left and right minimal indices of $\lambda E-A$, respectively. The matrix pencil $\lambda E-A, E, A \in \mathbb{C}^{n, m}$ is called regular if $n=m$ and $\operatorname{det}\left(\lambda_{0} E-A\right) \neq 0$ for some $\lambda_{0} \in \mathbb{C}$, otherwise it is called singular. A pencil is singular if and only if it has blocks of at least one of the types $\mathcal{L}_{\varepsilon_{j}}$ or $\mathcal{L}_{\eta_{j}}^{\top}$ in the Kronecker canonical form.

The values $\lambda_{1}, \ldots, \lambda_{r} \in \mathbb{C}$ are called the finite eigenvalues of $\lambda E-A$. If $s>0$, then $\lambda_{0}=\infty$ is said to be an eigenvalue of $\lambda E-A$. (Equivalently, zero is then an eigenvalue of 
the reversal $\lambda A-E$ of the pencil $\lambda E-A$.) The sum of all sizes of blocks that are associated with a fixed eigenvalue $\lambda_{0} \in \mathbb{C} \cup\{\infty\}$ is called the algebraic multiplicity of $\lambda_{0}$. The size of the largest block $\mathcal{N}_{\sigma_{j}}$ is called the index $\nu$ of the pencil $\lambda E-A$, where, by convention, $\nu=0$ if $E$ is invertible. The pencil is called stable if it is regular and if all eigenvalues are in the closed left half plane, and the ones lying on the imaginary axis (including infinity) have the largest associated block of size at most one. Otherwise the pencil is called unstable.

The following result was shown in [30]. We state the result in full generality, but clearly all statements also hold for the special case that $E, Q, J, R$ are real and that $Q=I$ which is the case considered in this paper.

Theorem 2 Let $E, Q \in \mathbb{C}^{n, m}$ satisfy $E^{H} Q=Q^{H} E \geq 0$ and let all left minimal indices of $\lambda E-Q$ be equal to zero (if there are any). Furthermore, let $J, R \in \mathbb{R}^{m, m}$ be such that we have $J=-J^{H}, R \geq 0$. Then the following statements hold for the pencil $L(\lambda)=\lambda E-(J-R) Q$.

(i) If $\lambda_{0} \in \mathbb{C}$ is an eigenvalue of $L(\lambda)$ then $\operatorname{Re}\left(\lambda_{0}\right) \leq 0$.

(ii) If $\omega \in \mathbb{R} \backslash\{0\}$ and $\lambda_{0}=i \omega$ is an eigenvalue of $L(\lambda)$, then $\lambda_{0}$ is semisimple. Moreover, if the columns of $V \in \mathbb{C}^{m, k}$ form a basis of a regular deflating subspace of $L(\lambda)$ associated with $\lambda_{0}$, then $R Q V=0$.

If, additionally, $Q$ is nonsingular then the previous statement holds for $\lambda_{0}=0$ as well. If $Q$ is singular then $\lambda_{0}=0$ need not be semisimple, but if $L(\lambda)$ is regular, then Jordan blocks associated with $\lambda_{0}=0$ have size at most two.

(iii) The index of $L(\lambda)$ is at most two.

(iv) All right minimal indices of $L(\lambda)$ are at most one (if there are any).

(v) If in addition $\lambda E-Q$ is regular, then all left minimal indices of $L(\lambda)$ are zero (if there are any).

Proof. For the proof see [30]. The additional statement in (ii) on the eigenvalue $\lambda_{0}=0$ was not presented in [30, but it follows in a straightforward manner from [30, Theorem 6.1] and the proof of [30, Corollary 6.2].

Theorem 2 illustrates that the special structure of $\mathrm{dH}$ systems imposes many restrictions in the spectral data and this has also an advantage when determining the distances to the nearest 'bad' problem. In particular, Theorem 2 implies that the distance to instability and the distance to higher index coincide for a pencil $L(\lambda)$ with $Q$ nonsingular.

The following well-known lemma, see [6, 23] (also stated for the general complex case), will be needed in order to make statements about the index of a matrix pencil in special situations.

Lemma 3 Let $E, A \in \mathbb{C}^{n, n}$ be matrices of the form

$$
E=\left[\begin{array}{cc}
E_{11} & 0 \\
0 & 0
\end{array}\right] \text { and } A=\left[\begin{array}{cc}
A_{11} & A_{12} \\
A_{21} & A_{22}
\end{array}\right]
$$

where $E_{11}$ is invertible.

(i) If $A_{22}$ is invertible, then the pencil $\lambda E-A$ is regular and has index one;

(ii) if $A_{22}$ is singular, then the pencil $\lambda E-A$ is singular or has an index greater than or equal to two. 


\section{Problem statement and main results for dHDAE systems}

We are interested in the following distance problems for matrix pencils $L(\lambda)$ of the form (3) under perturbations that preserve the special structure of the pencil.

Definition 4 Let $\mathcal{L}$ denote the class of square $n \times n$ real matrix pencils of the form (3). Then

1) the structured distance to singularity is defined as

$$
d_{\text {sing }}^{\mathcal{L}}(L(\lambda)):=\inf \left\{\left\|\Delta_{L}(\lambda)\right\|_{F} \mid L(\lambda)+\Delta_{L}(\lambda) \in \mathcal{L} \text { and is singular }\right\}
$$

2) the structured distance to the nearest high-index problem is defined as

$$
d_{\mathrm{hi}}^{\mathcal{L}}(L(\lambda)):=\inf \left\{\left\|\Delta_{L}(\lambda)\right\|_{F} \mid L(\lambda)+\Delta_{L}(\lambda) \in \mathcal{L} \text { and is of index } \geq 2\right\} ;
$$

3) the structured distance to instability is defined as

$$
d_{\text {inst }}^{\mathcal{L}}(L(\lambda)):=\inf \left\{\left\|\Delta_{L}(\lambda)\right\|_{F} \mid L(\lambda)+\Delta_{L}(\lambda) \in \mathcal{L} \text { and is unstable }\right\} .
$$

Note that all defined distances are meaningful, as for each matrix $X \in \mathbb{R}^{n, n}$ the decomposition into a sum $X=X_{1}+X_{2}$ of a skew-symmetric matrix $X_{1}=\frac{1}{2}\left(X-X^{\top}\right)$ and symmetric matrix $X_{2}=\frac{1}{2}\left(X+X^{\top}\right)$ is unique. Furthermore, we have $\|X\|_{F}^{2}=\left\|X_{1}\right\|_{F}^{2}+\left\|X_{2}\right\|_{F}^{2}=\left\|\left[X_{1}, X_{2}\right]\right\|_{F}^{2}$ due to the trace of $X_{1}^{\top} X_{2}$ being zero. Thus, the constraint $L(\lambda)+\Delta_{L}(\lambda) \in \mathcal{L}$ in (6) -(8) is the same as writing

$$
\Delta_{L}(\lambda)=\lambda \Delta_{E}-\left(\Delta_{J}-\Delta_{R}\right),
$$

with $\Delta_{J}=-\Delta_{J}^{\top}$ and $E+\Delta_{E}, R+\Delta_{R} \geq 0$, and we have $\left\|\left[\Delta_{J}, \Delta_{R}, \Delta_{E}\right]\right\|_{F}=\left\|\left[\Delta_{L}(\lambda)\right]\right\|_{F}$. The positivity conditions for $E+\Delta_{E}, R+\Delta_{R}$ are crucial. Examples presented in Section 5.2 show that they can neither be omitted nor simplified to $E+\Delta_{E}, R+\Delta_{R}$ being merely symmetric.

Theorem 5 Let $L(\lambda)=\lambda E-(J-R) \in \mathcal{L}$. Then the following statements hold.

(i) The pencil $L(\lambda)$ is singular if and only if ker $J \cap \operatorname{ker} E \cap \operatorname{ker} R \neq\{0\}$. In that case there exists an orthogonal transformation matrix $U \in \mathbb{R}^{n, n}$ such that

$$
U^{\top} E U=\left[\begin{array}{cc}
E_{11} & 0 \\
0 & 0
\end{array}\right], \quad U^{\top} J U=\left[\begin{array}{cc}
J_{11} & 0 \\
0 & 0
\end{array}\right], \quad U^{\top} R U=\left[\begin{array}{cc}
R_{11} & 0 \\
0 & 0
\end{array}\right],
$$

where the pencil $\lambda E_{11}-\left(J_{11}-R_{11}\right)$ is regular and has the size $(n-r) \times(n-r)$ with $r=\operatorname{dim} \operatorname{ker}(E-J+R)>0$. In particular, all right and left minimal indices of $L(\lambda)$ in its Kronecker canonical form are zero.

(ii) The index of $L(\lambda)$ is at most two. Furthermore, the following statements are equivalent.

(a) For any $\varepsilon>0$ there exists a pencil $\lambda \widetilde{E}-(\widetilde{J}-\widetilde{R})$ with $\widetilde{E}, \widetilde{R} \geq 0$, and $\widetilde{J}=-\widetilde{J}^{\top}$ which is regular and of index two such that

$$
\|[E-\widetilde{E}, J-\widetilde{J}, R-\widetilde{R}]\|_{F}=\|[E-\widetilde{E},(J-R)-(\widetilde{J}-\widetilde{R})]\|_{F} \leq \varepsilon,
$$

i.e., $L(\lambda)$ is in the closure of the set of regular $d H$ pencils of index two. 
(b) $\operatorname{ker} E \cap \operatorname{ker} R \neq\{0\}$.

To construct the perturbations where the distance to singularity ia achieved, we use the following ansatz. For a matrix $Y \in \mathbb{R}^{n, n}$ and a vector $u \in \mathbb{R}^{n}$ with $\|u\|_{2}=1$ we define the matrix

$$
\Delta_{Y}^{u}=-u u^{\top} Y-Y u u^{\top}+u u^{\top} Y u u^{\top},
$$

that will be used at several occasions during the paper. Then we obtain the following characterization of the distance to singularity.

Theorem 6 Let $\lambda E-(J-R) \in \mathcal{L}$. Then the following statements hold.

(i) The distance to singularity (6) is attained with a perturbation $\Delta_{E}=\Delta_{E}^{u}, \Delta_{J}=\Delta_{J}^{u}$, and $\Delta_{R}=\Delta_{R}^{u}$ as in (10) for some $u \in \mathbb{R}^{n}$ with $\|u\|_{2}=1$. The distance is given by

$$
\begin{aligned}
& d_{\text {sing }}^{\mathcal{L}}(\lambda E-(J-R)) \\
& =\min _{\substack{u \in \mathbb{R}^{n} \\
\|u\|=1}} \sqrt{2\|J u\|^{2}+2\left\|\left(I-u u^{\top}\right) E u\right\|^{2}+\left(u^{\top} E u\right)^{2}+2\left\|\left(I-u u^{\top}\right) R u\right\|^{2}+\left(u^{\top} R u\right)^{2}}
\end{aligned}
$$

and is bounded as

$$
\sqrt{\lambda_{\min }\left(-J^{2}+R^{2}+E^{2}\right)} \leq d_{\text {sing }}^{\mathcal{L}}(\lambda E-(J-R)) \leq \sqrt{2 \cdot \lambda_{\min }\left(-J^{2}+R^{2}+E^{2}\right)} .
$$

(ii) The distance to higher index (7) and the distance to instability (8) coincide and satisfy

$$
\begin{aligned}
d_{\mathrm{hi}}^{\mathcal{L}}(\lambda E-(J-R)) & =d_{\text {inst }}^{\mathcal{L}}(\lambda E-(J-R)) \\
& =\min _{\substack{u \in \mathbb{R}^{n} \\
\|u\|=1}} \sqrt{2\left\|\left(I-u u^{\top}\right) E u\right\|^{2}+\left(u^{\top} E u\right)^{2}+2\left\|\left(I-u u^{\top}\right) R u\right\|^{2}+\left(u^{\top} R u\right)^{2}}
\end{aligned}
$$

and are bounded as

$$
\sqrt{\lambda_{\min }\left(E^{2}+R^{2}\right)} \leq d_{\mathrm{hi}}^{\mathcal{L}}(\lambda E-(J-R))=d_{\text {inst }}^{\mathcal{L}}(\lambda E-(J-R)) \leq \sqrt{2 \cdot \lambda_{\min }\left(E^{2}+R^{2}\right)} .
$$

The proofs of Theorems [5 and 6] are given in Section 5.1, where they are obtained as simple consequences of a general theory developed in Section 4.2 for matrix polynomials with a special symmetry structure. Before we give the proofs, we will first consider a more general minimization problem in the next section.

\section{General distance problems}

In this section, we present a solution to a quite general minimization problem. This will allow us to solve the distance problems for $\mathrm{dH}$ pencils introduced in Section 3 as well as analogous problems for structured matrix polynomials with a $\mathrm{dH}$ like structure in a unified manner.

Theorem 5 states that both the distance to singularity as well as to higher index for a $\mathrm{dH}$ pencil as in (3) can be expressed via the existence of a common kernel of two or three structured matrices, so that both problems can be reinterpreted as a distance problem to the common kernel of matrices with symmetry and positivity structures. This concept will now be extended to more than three matrices. 


\subsection{Distance to the common kernel of a tuple of structured matrices}

Definition 7 Let $\mathcal{S}_{\ell}^{n}$ denote the following set of $(\ell+2)$-tuples of $n \times n$ real matrices

$$
\mathcal{S}_{\ell}^{n}:=\left\{\left(J, X_{0}, \ldots, X_{\ell}\right) \in\left(\mathbb{R}^{n, n}\right)^{\ell+2} \mid J^{\top}=-J, X_{i}=X_{i}^{T} \geq 0, i=1, \ldots, \ell\right\},
$$

where $\ell \geq 0$ and $n \geq 1$ are fixed. For a given tuple $\left(J, X_{0}, \ldots, X_{\ell}\right) \in \mathcal{S}_{\ell}^{n}$ we define the structured distance to the common kernel $d_{\mathrm{ker}}^{\mathcal{S}_{\ell}^{n}}\left(J, X_{0}, \ldots, X_{\ell}\right)$ as

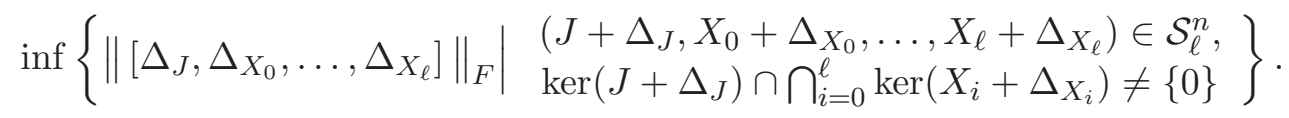

In the following, we often drop the dependence on $\ell$ and $n$ in the notation for simplicity, thus writing $d_{\mathrm{ker}}^{\mathcal{S}}\left(J, X_{0}, \ldots, X_{\ell}\right)$.

Observe that in determining $d_{\mathrm{ker}}^{\mathcal{S}}\left(J, X_{0}, \ldots, X_{\ell}\right)$ we measure the distance to a closed set.

Lemma 8 The set of all $\left(J, X_{0}, \ldots, X_{\ell}\right) \in \mathcal{S}_{\ell}^{n}$ satisfying $\operatorname{ker} J \cap \operatorname{ker} X_{0} \cap \cdots \cap \operatorname{ker} X_{\ell} \neq\{0\}$ is a closed subset in $\mathcal{S}_{\ell}^{n}$.

Proof. The proof follows by considering sequences of tuples $\left(J^{(m)}, X_{0}^{(m)}, \ldots, X_{\ell}^{(m)}\right)$ and a convergent subsequence of a sequence of unit vectors $u_{m}$ satisfying

$$
J^{(m)} u_{m}=X_{i}^{(m)} u_{m}=0, \quad i=1, \ldots \ell .
$$

Before we present the solution of the minimization problem, we first develop equivalent conditions for $J, X_{0}, \ldots, X_{\ell}$ to have a nontrivial common kernel.

Proposition 9 Let $\left(J, X_{0}, \ldots, X_{\ell}\right) \in \mathcal{S}_{\ell}^{n}$. Then

$\operatorname{ker} J \cap \operatorname{ker} X_{0} \cap \ldots \operatorname{ker} X_{\ell}=\operatorname{ker}\left(J^{\top} J+X_{0}^{2}+\cdots+X_{\ell}^{2}\right)=\operatorname{ker}\left(-J+X_{0}+\cdots+X_{\ell}\right)$.

Furthermore, there exists an orthogonal matrix $U \in \mathbb{R}^{n, n}$ such that

$$
U^{\top} J U=\left[\begin{array}{cc}
\widetilde{J} & 0 \\
0 & 0
\end{array}\right], \quad U^{\top} X_{i} U=\left[\begin{array}{cc}
\widetilde{X}_{i} & 0 \\
0 & 0
\end{array}\right], \quad i=0, \ldots, \ell
$$

with some $\widetilde{J}, \widetilde{X}_{0}, \ldots, \widetilde{X}_{\ell} \in \mathbb{R}^{n-r, n-r}$, where $r=\operatorname{dim} \operatorname{ker}\left(-J+X_{0}+\cdots+X_{\ell}\right) \geq 0$ and where the matrix $-\widetilde{J}+\widetilde{X}_{0}+\cdots+\widetilde{X}_{\ell}$ is invertible.

Proof. The inclusion ker $J \cap \operatorname{ker} X_{0} \cap \ldots \operatorname{ker} X_{\ell} \subseteq \operatorname{ker}\left(J^{\top} J+X_{0}^{2}+\cdots+X_{\ell}^{2}\right)$ is trivial. To prove the converse, let $x \in \operatorname{ker}\left(J^{T} J+X_{0}^{2}+\cdots+X_{\ell}^{2}\right)$ be nonzero. Since each summand is positive semidefinite, we obtain $X_{0}^{2} x=\cdots=X_{\ell}^{2} x=0$ and $J^{2} x=-J^{T} J x=0$. Noting that $\operatorname{ker} Y=\operatorname{ker} Y^{2}$ holds for any symmetric or skew-symmetric matrix finishes the proof.

The inclusion ker $J \cap \operatorname{ker} X_{0} \cap \ldots \operatorname{ker} X_{\ell} \subseteq \operatorname{ker}\left(-J+X_{0}+\cdots+X_{\ell}\right)$ is again trivial. To prove the converse let $x \in \operatorname{ker}\left(-J+X_{0}+\cdots+X_{\ell}\right)$ be nonzero. Since $x^{\top} J x=0$, we obtain that $x^{\top} X_{0} x+\cdots+x^{\top} X_{\ell} x=0$ and since each of the matrices $X_{0}, \ldots, X_{\ell}$ is positive semidefinite, we obtain $X_{0} x=\cdots=X_{\ell} x=0$, which then implies $J x=0$ as well.

To prove the last assertion, let $U$ be an orthogonal matrix with last $r$ columns spanning the kernel of $-J+X_{0}+\cdots+X_{\ell}$. Note that if $u$ is one of those $r$ last columns of $U$ then (13) implies that $u^{\top} J=0$ and $u^{\top} X_{i}=0$ for $i=0, \ldots, \ell$, which shows the formula (14). 
Remark 10 We highlight that the nonegativity assumption for the matrices $X_{i}$ is crucial for the two nontrivial inclusions in Proposition 9, For example, consider

$$
J=\left[\begin{array}{cc}
0 & 1 \\
-1 & 0
\end{array}\right] \text { and } X_{0}=\left[\begin{array}{cc}
1 & 0 \\
0 & -1
\end{array}\right]
$$

Then $J-X_{0}$ is singular while the intersection of the kernels of $J$ and $X_{0}$ is trivial.

Also note that while an arbitrarily large number of symmetric positive semidefinite matrices $X_{0}, \ldots, X_{\ell}$ can be considered, the results from Proposition 9 are no longer true if a second skew-symmetric matrix is involved. For example, consider the matrices

$$
J_{1}=\left[\begin{array}{ccc}
0 & 1 & 0 \\
-1 & 0 & 0 \\
0 & 0 & 0
\end{array}\right] \quad \text { and } J_{2}=\left[\begin{array}{ccc}
0 & 0 & 1 \\
0 & 0 & 0 \\
-1 & 0 & 0
\end{array}\right]
$$

Then $J_{1}+J_{2}$ is singular (in fact, even the pencil $\lambda J_{1}+J_{2}$ is singular), but $J_{1}$ and $J_{2}$ do not have a common kernel.

Given $\left(J, X_{0}, \ldots, X_{\ell}\right) \in \mathcal{S}_{\ell}^{n}$ as in Proposition 9. we aim to characterize all perturbations that produce a nontrivial common kernel of the matrices $J, X_{0}, \ldots, X_{\ell}$ while preserving their individual structures. For this, we will use particular perturbations whose special properties will be presented in the following lemma.

Lemma 11 Let $Y \in \mathbb{R}^{n, n}$, let $u \in \mathbb{R}^{n, n}$ be a vector with $\|u\|_{2}=1$ and let

$$
\Delta_{Y}^{u}:=-u u^{\top} Y-Y u u^{\top}+u u^{\top} Y u u^{\top} .
$$

Then the following statements hold.

(i) $u \in \operatorname{ker}\left(Y+\Delta_{Y}^{u}\right)$, in particular, $Y+\Delta_{Y}^{u}$ is singular.

(ii) rank $\Delta_{Y}^{u} \leq 2$, and rank $\Delta_{Y}^{u} \leq 1$ if and only if $u$ is a right or left eigenvector of $Y$.

(iii) $\left\|\Delta_{Y}^{u}\right\|_{F}^{2}=\left\|\left(I-u u^{\top}\right) Y u\right\|_{F}^{2}+\left\|u^{\top} Y\left(I-u u^{\top}\right)\right\|_{F}^{2}+\left(u^{\top} Y u\right)^{2}$.

(iv) If $Y \geq 0$ then $Y+\Delta_{Y}^{u} \geq 0$ and $\left\|\Delta_{Y}^{u}\right\|_{F}^{2}=2\left\|\left(I-u u^{\top}\right) Y u\right\|_{2}^{2}+\left(u^{\top} Y u\right)^{2}$.

(v) If $Y^{\top}=-Y$, then $\Delta_{Y}^{u}=-u u^{\top} Y-Y u u^{\top}$ and $\left\|\Delta_{Y}^{u}\right\|_{F}^{2}=2\|Y u\|_{2}$.

Proof. (ii) immediately follows from $\Delta_{Y}^{u} u=-Y u$. For the proof of (iii) let $U \in \mathbb{R}^{n, n}$ be an orthogonal matrix with last column $u$. Then we obtain

$$
U^{T} Y U=\left[\begin{array}{ll}
Y_{11} & Y_{12} \\
Y_{21} & Y_{22}
\end{array}\right] \text { and } U^{T} \Delta_{Y}^{u} U=\left[\begin{array}{cc}
0 & -Y_{12} \\
-Y_{21} & -Y_{22}
\end{array}\right]
$$

for some $Y_{11}, Y_{12}, Y_{21}, Y_{22}$ with $Y_{11} \in \mathbb{R}^{n-1, n-1}$ which immediately shows that rank $\Delta_{Y}^{u} \leq 2$. In particular, we have $\operatorname{rank} \Delta_{Y}^{u} \leq 1$ if and only if $Y_{12}=0$ or $Y_{21}=0$ which is equivalent to $u$ being a right or left eigenvector of $Y$, respectively. Moreover, (iii) immediately follows from the representation (16) using that

$$
\left(I-u u^{\top}\right) Y u=\left[\begin{array}{c}
Y_{12} \\
0
\end{array}\right], \quad u Y\left(I-u u^{\top}\right)=\left[\begin{array}{ll}
Y_{21} & 0
\end{array}\right], \quad \text { and } \quad Y_{22}=u^{\top} Y u
$$


Finally, using the additional (skew-)symmetry structure, we obtain (iv) and (v), where the part $Y+\Delta_{Y}^{u} \geq 0$ in (iv) again follows from the representation (16).

We highlight that the first property of statement (iv) in Lemma 11] will become essential in what follows, because it allows us to perform a perturbation that makes a symmetric matrix singular while simultaneously preserving the positive semidefiniteness of the matrix. With these preparations, we obtain the following theorem that characterizes structure-preserving perturbations to matrices with a nontrivial common kernel.

Theorem 12 Let $\left(J, X_{0}, \ldots, X_{\ell}\right) \in \mathcal{S}_{\ell}^{n}$, i.e., $J^{\top}=-J$ and $X_{i}^{\top}=X_{i} \geq 0$ for $i=0, \ldots, \ell$. Furthermore, for any $u \in \mathbb{R}^{n},\|u\|_{2}=1$, consider the perturbation matrices

$$
\Delta_{J}^{u}:=-u u^{\top} J-J u u^{\top} \quad \text { and } \quad \Delta_{X_{i}}^{u}:=-u u^{\top} X_{i}-X_{i} u u^{\top}+u u^{\top} X_{i} u u^{\top}, \quad i=0, \ldots, \ell .
$$

Then the following statements hold.

(i) For any vector $u \in \mathbb{R}^{n}$, $\|u\|_{2}=1$, we have

$$
\left(\Delta_{J}^{u}\right)^{\top}=-\Delta_{J}^{u}, \quad \text { as well as }\left(\Delta_{X_{i}}^{u}\right)^{\top}=\Delta_{X_{i}}^{u} \text { and } X_{i}+\Delta_{X_{i}}^{u} \geq 0, \quad i=0, \ldots, \ell .
$$

Furthermore, the kernels of the matrices $J+\Delta_{J}^{u}, X_{0}+\Delta_{X_{0}}^{u}, \ldots, X_{\ell}+\Delta_{X_{\ell}}^{u}$ have a nontrivial intersection.

(ii) For any vector $u \in \mathbb{R}^{n},\|u\|_{2}=1$, we have

$$
\left\|\Delta_{J}^{u}\right\|_{F}^{2}=2\|J u\|_{2}, \quad \text { and } \quad\left\|\Delta_{X_{i}}^{u}\right\|_{F}^{2}=2\left\|\left(I-u u^{\top}\right) X_{i} u\right\|^{2}+\left(u^{\top} X_{i} u\right)^{2}, \quad i=0, \ldots, \ell .
$$

(iii) Let $\Delta_{J}, \Delta_{X_{0}}, \ldots, \Delta_{X_{\ell}} \in \mathbb{R}^{n, n}$ be any perturbation matrices satisfying

$$
\Delta_{J}^{\top}=-\Delta_{J} \quad \text { as well as } \Delta_{X_{i}}^{\top}=\Delta_{X_{i}}, \text { and } X_{i}+\Delta_{X_{i}} \geq 0, \quad i=0, \ldots, \ell,
$$

and such that the kernels of the matrices $J+\Delta_{J}, X_{0}+\Delta_{X_{0}}, \ldots, X_{\ell}+\Delta_{X_{\ell}}$ have a nontrivial intersection. Then

$$
\left\|\Delta_{J}^{u}\right\|_{F} \leq\left\|\Delta_{J}\right\|_{F}, \quad \text { and } \quad\left\|\Delta_{X_{i}}^{u}\right\|_{F} \leq\left\|\Delta_{X_{i}}\right\|_{F}, \quad i=0, \ldots, \ell .
$$

for some real vector $u$ with $\|u\|_{2}=1$

Proof. (iil) and (iii) follow immediately from Lemma 11. To prove (iii), consider any perturbation matrices $\Delta_{J}, \Delta_{X_{0}}, \ldots, \Delta_{X_{\ell}}$ satisfying (19) such that the kernels of the matrices $J+\Delta_{J}, X_{0}+\Delta_{X_{0}}, \ldots, X_{\ell}+\Delta_{X_{\ell}}$ have a nontrivial intersection. Then by Proposition 9 , there exists an orthogonal matrix $U$ such that

$$
U^{\top}\left(J+\Delta_{J}\right) U=\left[\begin{array}{cc}
\widetilde{J} & 0 \\
0 & 0
\end{array}\right], \quad U^{\top}\left(X_{i}+\Delta_{X_{i}}\right) U=\left[\begin{array}{cc}
\tilde{X}_{i} & 0 \\
0 & 0
\end{array}\right], \quad i=0, \ldots, \ell
$$

with some $\widetilde{J}, \widetilde{X}_{0}, \ldots, \widetilde{X}_{\ell} \in \mathbb{R}^{n-1, n-1}$, not necessarily invertible, i.e., in contrast to (14) we split only one vector from the intersection of kernels. Transforming and decomposing accordingly, we have

$$
U^{\top} J U=\left[\begin{array}{cc}
\widetilde{K} & t \\
-t^{\top} & 0
\end{array}\right], \quad \text { and } \quad U^{\top} X_{i} U=\left[\begin{array}{cc}
\widetilde{S}_{i} & s_{i} \\
s_{i}^{\top} & r_{i}
\end{array}\right], \quad i=0, \ldots, \ell
$$


for some skew-symmetric matrix $\widetilde{K} \in \mathbb{R}^{n-1, n-1}$, some symmetric matrices $\widetilde{S}_{i} \in \mathbb{R}^{n-1, n-1}$, some $r_{i} \in \mathbb{R}, s_{i} \in \mathbb{R}^{n-1}(i=0, \ldots, \ell)$, and some $t \in \mathbb{R}^{n-1}$. Subtracting (21) from (20), we obtain that

$$
U^{\top} \Delta_{J} U=\left[\begin{array}{cc}
\widetilde{J}-\widetilde{K} & -t \\
t^{\top} & 0
\end{array}\right], \quad \text { and } \quad U^{\top} \Delta_{X_{i}} U=\left[\begin{array}{cc}
\widetilde{X}_{i}-\widetilde{S}_{i} & -s_{i} \\
-s_{i}^{\top} & -r_{i}
\end{array}\right], \quad i=0, \ldots, \ell .
$$

Observe that for the particular choice $u=U e_{n}$ the perturbations from (15) have, by (21), the forms

$$
U^{\top} \Delta_{J}^{u} U=-\left[\begin{array}{cc}
0 & -t \\
t^{\top} & 0
\end{array}\right] \quad \text { and } \quad U^{\top} \Delta_{X_{i}}^{u} U=-\left[\begin{array}{cc}
0 & s_{i} \\
s_{i}^{\top} & r_{i}
\end{array}\right], \quad i=0, \ldots, \ell .
$$

Since the Frobenius norm is invariant under real orthogonal transformations, we immediately obtain that $\left\|\Delta_{J}^{u}\right\|_{F} \leq\left\|\Delta_{J}\right\|_{F}$ and $\left\|\Delta_{X_{i}}^{u}\right\|_{F} \leq\left\|\Delta_{X_{i}}\right\|_{F}$ for $i=0, \ldots, \ell$.

We now have all ingredients to state and prove the solution of our general minimization problem.

Theorem 13 Let $\left(J, X_{0}, \ldots, X_{\ell}\right) \in \mathcal{S}_{\ell}^{n}$, i.e., $J^{\top}=-J$ and $X_{j}^{\top}=X_{j} \geq 0$ for $j=1, \ldots, \ell$. Then the structured distance $d_{\mathrm{ker}}^{\mathcal{S}}\left(J, X_{0}, \ldots, X_{\ell}\right)$ to the common kernel (12) is attained at $\Delta_{J}=\Delta_{J}^{u}, \Delta_{X_{0}}=\Delta_{X_{0}}^{u}, \ldots, \Delta_{X_{\ell}}=\Delta_{X_{\ell}}^{u}$ being as in (17) for some $u \in \mathbb{R}^{n}$ with $\|u\|_{2}=1$. Consequently,

$$
d_{\mathrm{ker}}^{\mathcal{S}}\left(J, X_{0}, \ldots, X_{\ell}\right)=\min _{u \in \mathbb{R}^{n},\|u\|=1}\left(2\|J u\|_{2}+\sum_{i=1}^{\ell}\left(2\left\|\left(I-u u^{\top}\right) X_{i} u\right\|^{2}+\left(u^{\top} X_{i} u\right)^{2}\right)\right)^{1 / 2}
$$

and in addition, we have the bounds

$$
\sqrt{\lambda_{\min }\left(-J^{2}+X_{0}^{2}+\cdots+X_{\ell}^{2}\right)} \leq d_{\mathrm{ker}}^{\mathcal{S}}\left(J, X_{0}, \ldots, X_{\ell}\right) \leq \sqrt{2 \cdot \lambda_{\min }\left(-J^{2}+X_{0}^{2}+\cdots+X_{\ell}^{2}\right)} .
$$

Proof. The first two statements follow directly from Theorem 12. It remains to prove (24). For this aim note that for every $u \in \mathbb{R}^{n}$ with $\|u\|_{2}=1$, we have

$$
\begin{aligned}
u^{\top}\left(-J^{2}+X_{0}^{2}+\cdots+X_{\ell}^{2}\right) u & =\|J u\|^{2}+\left\|X_{0} u\right\|^{2}+\cdots+\left\|X_{\ell} u\right\|^{2} \\
& =\left(\|J u\|^{2}+\sum_{i=1}^{\ell}\left(\left\|\left(I-u u^{\top}\right) X_{i} u\right\|^{2}+\left(u^{\top} X_{i} u\right)^{2}\right)\right) .
\end{aligned}
$$

Taking the infimum over all $u \in \mathbb{R}^{n}$ with $\|u\|_{2}=1$ shows (24).

Remark 14 In the special case $\lambda_{\min }\left(-J^{2}+X_{0}^{2}+\cdots+X_{\ell}^{2}\right)=0$ it immediately follows that $d_{\text {ker }}^{\mathcal{S}}\left(J, X_{0}, \ldots, X_{\ell}\right)=0$. This is in line with Proposition 9 , because the singularity of the matrix $-J^{2}+X_{0}^{2}+\cdots+X_{\ell}^{2}$ is equivalent to the existence of a nontrivial common kernel of the matrices $J, X_{0}, \ldots, X_{\ell}$. 


\subsection{Distance problems for structured matrix polynomials}

As a first application of the results from Subsection 4.1, we will consider distance problems for a particular class of structured matrix polynomials. To this end, recall from [15 that by definition a square matrix polynomial $P(\lambda)=\sum_{i=0}^{k} \lambda^{i} Y_{i}$ is singular if and only if $\operatorname{det} P(\lambda) \equiv 0$. Also recall that the companion linearization

$$
L(\lambda)=\lambda\left[\begin{array}{llll}
Y_{k} & & & \\
& I & & \\
& & \ddots & \\
& & & I
\end{array}\right]+\left[\begin{array}{cccc}
Y_{k-1} & \ldots & Y_{1} & Y_{0} \\
-I & 0 & & \\
& \ddots & \ddots & \\
& & -I & 0
\end{array}\right] .
$$

of $P(\lambda)$ is a strong linearisation in the sense of [10]. In particular, $L(\lambda)$ is singular if and only if $P(\lambda)$ is singular. Furthermore, as shown in [10, in the linearization the spectral data for eigenvalues of $P(\lambda)$ is preserved. Therefore, for the sake of simplicity, we define the notions of index and instability for the matrix polynomial $P(\lambda)$ via the respective notions of the Kronecker canonical form of (25), cf. Section 2. We then extend Definition 4 as follows.

Definition 15 Consider the class of matrix polynomials

$$
\mathcal{P}_{k, j}^{n}:=\left\{-\lambda^{j} J+\sum_{i=0}^{k} \lambda^{i} A_{i} \mid J^{\top}=-J, A_{i}^{\top}=A_{i} \geq 0 \in \mathbb{R}^{n, n}, i=0, \ldots, k\right\},
$$

where $n \geq 1, k, j \geq 0$ and, without loss of generality, $j \leq k$. Then for $P(\lambda) \in \mathcal{P}_{k, j}^{n}$

1) the structured distance to singularity is defined as

$$
d_{\text {sing }}^{\mathcal{P}_{k, j}^{n}}(P(\lambda)):=\inf \left\{\left\|\Delta_{P}(\lambda)\right\|_{F} \mid P(\lambda)+\Delta_{P}(\lambda) \in \mathcal{P}_{k, j}^{n} \text { is singular }\right\} ;
$$

2) the structured distance to the nearest high index problem is defined as

$$
d_{\mathrm{hi}}^{\mathcal{P}_{k, j}^{n}}(P(\lambda)):=\inf \left\{\left\|\Delta_{P}(\lambda)\right\|_{F} \mid P(\lambda)+\Delta_{P}(\lambda) \in \mathcal{P}_{k, j}^{n} \text { is of index } \geq 2\right\} ;
$$

3) the structured distance to instability is defined as

$$
d_{\text {inst }}^{\mathcal{P}_{k, j}^{n}}(P(\lambda)):=\inf \left\{\left\|\Delta_{P}(\lambda)\right\|_{F} \mid P(\lambda)+\Delta_{P}(\lambda) \in \mathcal{P}_{k, j}^{n} \text { is unstable }\right\} .
$$

We often simply write $d_{\star}^{\mathcal{P}}(P(\lambda))$ instead of $d_{\star}^{\mathcal{P}_{k, j}^{n}}(P(\lambda))$ for $\star \in\{$ sing, hi, inst $\}$.

In other words, $\mathcal{P}_{k, j}^{n}$ consists of the set of matrix polynomials of degree less than or equal to $k$ for which all coefficients are symmetric positive semidefinite except for the coefficient at $\lambda^{j}$ which is only assumed to have a positive semidefinite symmetric part. Particular examples for this kind of matrix polynomials are the $\mathrm{dH}$ pencils of the form (3), i.e., the set $\mathcal{P}_{1,0}^{n}$, and quadratic matrix polynomials of the form (4), i.e., the set $\mathcal{P}_{2,1}^{n}$. Observe that if both $P(\lambda), P(\lambda)+\Delta_{P}(\lambda) \in \mathcal{P}_{k, j}^{n}$ then $\Delta_{P}(\lambda)$ must take the form

$$
\Delta_{P}(\lambda)=-\lambda^{j} \Delta_{J}+\sum_{i=0}^{k} \lambda^{i} \Delta_{A_{i}}
$$

where $\Delta_{J}^{\top}=-\Delta_{J}$ and $\Delta_{A_{i}}^{\top}=\Delta_{A_{i}}$. We have the following theorem for characterizing the distance to the nearest singular or high index matrix polynomial. 
Theorem 16 Let $k \geq 1$ and $j \in\{0, \ldots, k\}$ and consider the set $P_{k, j}^{n}$ of matrix polynomials

$$
P(\lambda)=-\lambda^{j} J+\sum_{i=0}^{k} \lambda^{i} A_{i}
$$

with $J, A_{0}, \ldots, A_{k} \in \mathbb{R}^{n, n}, J^{\top}=-J$ and $A_{i}^{\top}=A_{i} \geq 0$ for $i=0, \ldots, k$.

(i) If $P(\lambda) \in \mathcal{P}_{k, j}^{n}$ then the following statements are equivalent:

(a) the polynomial $P(\lambda)$ is singular, i.e., $\operatorname{det} P(\lambda) \equiv 0$;

(b) the matrix $P(1)$ is singular;

(c) the kernels of the matrices $J, A_{0}, \ldots, A_{k}$ have a nontrivial intersection.

(ii) If $P(\lambda) \in \mathcal{P}_{k, j}^{n}$, then its distance to the set of singular matrix polynomials in $\mathcal{P}_{k, j}^{n}$ equals the distance to the common kernel of the matrices $J, A_{0}, \ldots, A_{k}$, i.e.,

$$
d_{\text {sing }}^{\mathcal{P}}(P(\lambda))=d_{\mathrm{ker}}^{\mathcal{S}}\left(J, A_{0}, \ldots, A_{k}\right)
$$

(iii) If $\max \{n, k\}>1$, then the closure of the set

$$
\mathcal{I}_{\text {hi }}:=\left\{P(\lambda) \in \mathcal{P}_{k, j}^{n} \mid P(\lambda) \text { is regular and has index greater than one }\right\}
$$

in $\mathcal{P}_{k, j}^{n}$ is equal to

$$
\mathcal{K}:=\left\{-\lambda^{j} J+\sum_{i=0}^{k} \lambda^{i} A_{i} \mid \operatorname{ker} A_{k} \cap \operatorname{ker} A_{k-1} \neq\{0\}\right\} \quad \text { if } j<k
$$

or to

$$
\mathcal{K}:=\left\{-\lambda^{k} J+\sum_{i=0}^{k} \lambda^{i} A_{i} \mid \operatorname{ker} J \cap \operatorname{ker} A_{k} \cap \operatorname{ker} A_{k-1} \neq\{0\}\right\} \quad \text { if } j=k>1 .
$$

If $\max \{n, k\}=1$ or $j=k=1$, then $\mathcal{I}_{\text {hi }}$ is empty.

(iv) Let $P(\lambda) \in \mathcal{P}_{k, j}^{n}$. If $\max \{n, k\}>1$, then the distance of $P(\lambda)$ to the set of higher index polynomials in $\mathcal{P}_{k, j}^{n}$ equals the distance to the respective common kernel

$$
d_{\mathrm{hi}}^{\mathcal{P}}(P(\lambda))= \begin{cases}d_{\mathrm{ker}}^{\mathcal{S}}\left(0, A_{k}, A_{k-1}\right) & \text { if } j<k \\ d_{\mathrm{ker}}^{\mathcal{S}}\left(J, A_{k}, A_{k-1}\right) & \text { if } j=k>1 .\end{cases}
$$

If $\max \{n, k\}=1$ or $j=k=1$, then $d_{\mathrm{hi}}^{\mathcal{P}}(P(\lambda))=\infty$.

Before we give the proof we make a few remarks.

Remark 17 We observe the following simple facts about the inclusions $\mathcal{P}_{k, j}^{n} \subseteq \mathcal{P}_{k+1, j}^{n}, k \geq 0$.

1) It is an immediate corollary from equation (29) that for $P(\lambda) \in \mathcal{P}_{k, j}^{n}$ one has

$$
d_{\text {sing }}^{\mathcal{P}_{k, j}^{n}}(P(\lambda))=d_{\text {sing }}^{\mathcal{P}_{\ell, j}^{n}}(P(\lambda)), \quad \ell \geq k .
$$


2) Observe that the closest singular polynomial may be of lower degree than the original one, e.g., let $\varepsilon>0$ be small and let

$$
P(\lambda)=\lambda\left[\begin{array}{ll}
\varepsilon & 0 \\
0 & 0
\end{array}\right]+\left[\begin{array}{ll}
0 & 0 \\
0 & 1
\end{array}\right] \in \mathcal{P}_{1,0}^{2} .
$$

Then the closest singular pencil in $\mathcal{P}_{1,0}^{2}$ is obtained by removing the $\varepsilon$ entry, and this is a pencil of degree zero.

3) The (algebraic, geometric, partial) multiplicities of the eigenvalue infinity and the index of a matrix polynomial $P(\lambda) \in \mathcal{P}_{k, j}^{n}$ are invariants with respect to the parameter $k$ and not with respect to the degree of the polynomial. For example consider the matrix polynomial $P(\lambda)=\sum_{i=0}^{k} \lambda^{i} A_{i} \in \mathcal{P}_{k, j}^{n}$ with $A_{0}=I_{n}$ and $A_{i}=0$ for $i=1, \ldots, k$ which is a matrix polynomial of degree zero. If $P(\lambda)$ is considered to be a matrix pencil (i.e. $k=1)$ then it is of index one and the algebraic multiplicity of $\infty$ is $n$. If, however, $P(\lambda)$ is considered as a quadratic matrix polynomial (i.e., $k=2$ ), then it companion linearization has the form

$$
\lambda\left[\begin{array}{cc}
0 & 0 \\
0 & I_{n}
\end{array}\right]+\left[\begin{array}{cc}
0 & I_{n} \\
-I_{n} & 0
\end{array}\right]
$$

and it follows that the eigenvalue $\infty$ has algebraic multiplicity $2 n$ and the index is two. The fact that a consistent spectral theory of matrix polynomials is only possible if the leading coefficients are allowed to be zero is a well-known fact in the theory of matrix polynomials (see [19]) and led to the introduction of the notion grade for the parameter $k$ in [26]. Consequently, there is in general no equality between $d_{\mathrm{hi}}^{\mathcal{P}_{k, j}^{n}}(P(\lambda))$ and $d_{\mathrm{hi}}^{\mathcal{P}_{\ell, j}^{n}}(P(\lambda))$ for $\ell>k$ which is also reflected by formula (32).

Proof. (1i) The implication (a) $\Rightarrow$ (b) is trivial. Next, if $P(1)=-J+A_{0}+\cdots+A_{k}$ is singular, then it follows from Proposition 9 that the kernels of $J, A_{0}, \ldots, A_{k}$ have a nontrivial intersection which, in turn, implies that $P(\lambda)$ is singular as obviously $\operatorname{det} P(\lambda) \equiv 0$. Then (iii) is an easy consequence of (1i).

(iii) First, consider the case $n=1$. If $k=1$, then $\mathcal{I}_{\text {hi }}$ is clearly empty. If $k>1$ and $P(\lambda) \in \mathcal{K}$, then $J=A_{k}=A_{k-1}=0$ and the companion form of $P(\lambda)$ is

$$
L(\lambda)=\lambda\left[\begin{array}{llll}
0 & & & \\
& 1 & & \\
& & \ddots & \\
& & & 1
\end{array}\right]+\left[\begin{array}{cccc}
0 & A_{k-2} & \ldots & A_{0} \\
-1 & 0 & & \\
& \ddots & \ddots & \\
& & -1 & 0
\end{array}\right]
$$

By Lemma 3 the index of $L(\lambda)$ and hence that of $P(\lambda)$ is at least two. If $P(\lambda)$ is regular, we thus have $P(\lambda) \in \mathcal{I}_{\mathrm{hi}}$. If $P(\lambda)$ is singular, then it is identically zero and replacing $A_{0}$ with $\varepsilon$ and letting $\varepsilon \rightarrow 0$, we see that $P(\lambda)$ is in the closure of $\mathcal{I}_{\text {hi }}$.

For $n>1$ we distinguish the cases $j<k$ and $j=k$.

Case $j<k$. First observe that $\mathcal{K}$ is a closed set in $\mathcal{P}_{k, j}^{n}$ by Lemma 8 . Hence, to prove the inclusion $\overline{\mathcal{I}}_{\text {hi }} \subseteq \mathcal{K}$ it suffices to show that any matrix polynomial $P(\lambda) \in \mathcal{I}_{\text {hi }}$ satisfies ker $A_{k} \cap \operatorname{ker} A_{k-1} \neq\{0\}$. To do this, suppose on the contrary that for some $P(\lambda) \in \mathcal{I}_{\text {hi }}$ we have ker $A_{k} \cap \operatorname{ker} A_{k-1}=\{0\}$. If ker $A_{k}=\{0\}$ then the matrix polynomial has no infinite 
eigenvalues and hence is of index zero. Hence, we may assume that $A_{k}$ has a nontrivial kernel, and then there exists an orthogonal congruence transformation so that

$$
U^{\top} A_{k} U=\left[\begin{array}{cc}
\widetilde{A}_{k} & 0 \\
0 & 0
\end{array}\right], U^{\top} J U=\left[\begin{array}{cc}
J_{11} & J_{12} \\
-J_{12}^{\top} & J_{22}
\end{array}\right], \quad \text { and } \quad U^{\top} A_{k-1} U=\left[\begin{array}{cc}
A_{11} & A_{12} \\
A_{12}^{\top} & A_{22}
\end{array}\right]
$$

where $\widetilde{A}_{k} \in \mathbb{R}^{n-r, n-r}(r>0)$ is invertible and all three matrices are partitioned conformably. In fact, replacing $P(\lambda)$ by $U^{\top} P(\lambda) U$ if necessary, we may assume $U=I_{n}$ in what follows. Since ker $A_{k} \cap \operatorname{ker} A_{k-1}=\{0\}$ and since $A_{k-1}$ is positive semidefinite, it then follows that $A_{22}$ is invertible.

If $j<k-1$ then the companion linearization (25) of $P(\lambda)$ takes the form

$$
\lambda\left[\begin{array}{cc|c}
\widetilde{A}_{k} & & \\
& 0 & \\
\hline & & I_{(k-1) n}
\end{array}\right]+\left[\begin{array}{cc|c}
A_{11} & A_{12} & * \\
A_{12}^{\top} & A_{22} & * \\
\hline * & * & *
\end{array}\right],
$$

where in comparison to (25) the first block row and column have been split into two, the last $k-1$ block rows and columns have been merged into one, respectively, and $*$ denotes a possibly nonzero block entry. Then it follows from Lemma 3 (applied to the pencil that is obtained from (34) by permuting the second and third block rows and columns) that the companion pencil and hence the matrix polynomial $P(\lambda)$ is of index one, since $A_{22}$ is invertible.

If $j=k-1$, then the coefficient of $\lambda^{k-1}$ in $P(\lambda)$ is given by $A_{k-1}-J$ and hence the companion linearization (25) of $P(\lambda)$ has the form

$$
\lambda\left[\begin{array}{cc|c}
\widetilde{A}_{k} & & \\
& 0 & \\
\hline & & I_{(k-1) n}
\end{array}\right]+\left[\begin{array}{cc|c}
A_{11}-J_{11} & A_{12}-J_{12} & * \\
A_{12}^{\top}+J_{12}^{\top} & A_{22}-J_{22} & * \\
\hline * & * & *
\end{array}\right]
$$

with the same conventions as for the pencil (34). But with $A_{22}$ invertible also $A_{22}-J_{22}$ is invertible (see Proposition 9 applied with $\ell=0$ to $J_{22}$ and $X_{0}=A_{22}$ ). Again, it follows from Lemma 3 that the matrix polynomial $P(\lambda)$ is of index one.

For the converse inclusion $\mathcal{K} \subseteq \overline{\mathcal{I}}_{\text {hi }}$ consider a matrix polynomial $P(\lambda) \in \mathcal{K}$. Furthermore, let $u \in \operatorname{ker} A_{k} \cap \operatorname{ker} A_{k-1}$ with $\|u\|_{2}=1$ and let $U \in \mathbb{R}^{n, n}$ be orthogonal with last column $u$. Then

$$
U^{\top} A_{k} U=\left[\begin{array}{cc}
\widetilde{A}_{k} & 0 \\
0 & 0
\end{array}\right], U^{\top} A_{k-1} U=\left[\begin{array}{cc}
\widetilde{A}_{k-1} & 0 \\
0 & 0
\end{array}\right], U^{\top} J U=\left[\begin{array}{cc}
J_{11} & v \\
-v^{\top} & 0
\end{array}\right],
$$

with $\widetilde{A}_{k}, \widetilde{A}_{k-1}, J_{11} \in \mathbb{R}^{n-1, n-1}$ (not necessarily being invertible) and $v \in \mathbb{R}^{n-1}$. Note that the entry 0 in $(2,2)$ block of $U^{\top} J U$ is caused by the skew-symmetry of $J$. Again, replacing $P(\lambda)$ with $U^{\top} P(\lambda) U$ if necessary, we may assume without loss of generality that $U=I_{n}$.

First assume that $j<k-1$. For small $\varepsilon>0$ we have that $\widetilde{A}_{k}+\varepsilon I_{n-1} \in \mathbb{R}^{n-1, n-1}$ and $A_{j}-J+\varepsilon I_{n} \in \mathbb{R}^{n, n}$ are invertible. Then the matrix polynomial $P_{\varepsilon}(\lambda)$ that is obtained from $P(\lambda)$ by replacing $A_{k}$ with $A_{k}+\operatorname{diag}\left(\varepsilon I_{n-1}, 0\right)$ and $A_{j}-J$ with $A_{j}-J+\varepsilon I_{n} \in \mathbb{R}^{n, n}$ is regular, because at least one coefficient (namely the coefficient associated with $\lambda^{j}$ ) is invertible. Furthermore, the companion linearization of $P_{\varepsilon}(\lambda)$ takes the form

$$
\lambda\left[\begin{array}{cc|c}
\widetilde{A}_{k}+\varepsilon I_{n-1} & & \\
& 0 & \\
\hline & I_{(k-1) n}
\end{array}\right]+\left[\begin{array}{cc|c}
\widetilde{A}_{k-1} & 0 & * \\
0 & 0 & * \\
\hline * & * & *
\end{array}\right] .
$$


By Lemma 3 we see that this pencil, and hence $P_{\varepsilon}(\lambda)$ itself, has index greater than one.

Now let $j=k-1$. For sufficiently small $\varepsilon>0$ we have that $\widetilde{A}_{k}+\varepsilon I_{n-1} \in \mathbb{R}^{n-1, n-1}$ and $A_{k-1}+\varepsilon I_{n} \in \mathbb{R}^{n, n}$ are invertible and that $v+\varepsilon e_{1} \neq 0$. Let $P_{\varepsilon}(\lambda)$ be the matrix polynomial obtained from $P(\lambda)$ by replacing $A_{k}$ and with $A_{k}+\operatorname{diag}\left(\varepsilon I_{n-1}, 0\right)$ and $A_{k-1}$ with $A_{k-1}+\varepsilon I_{n}$ as well as $v$ in $J$ with $v+\varepsilon e_{1} \neq 0$. Again, it follows that $P_{\varepsilon}(\lambda)$ is regular as the coefficient at $\lambda^{k-1}$ is regular (see Proposition 9 applied with $\ell=0$ to $J$ and $X_{0}=A_{k-1}+\varepsilon I_{n}$ ). Then the companion linearization of $P_{\varepsilon}(\lambda)$ takes the form

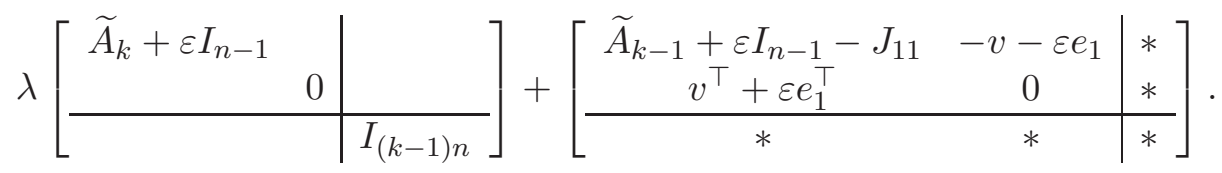

By Lemma 3 we see that (38), and hence $P_{\varepsilon}(\lambda)$ itself, has index greater than one.

Letting $\varepsilon \rightarrow 0$ we see that in both cases $j<k-1$ and $j=k-1$ we have $\mathcal{I}_{\text {hi }} \ni P_{\varepsilon}(\lambda) \rightarrow$ $P(\lambda) \in \overline{\mathcal{I}}_{\text {hi }}$.

Case $j=k$. If $j=k=1$ and $P(\lambda)=\lambda\left(J+A_{1}\right)+A_{0} \in \mathcal{P}_{1,1}^{n}$, then by Theorem 2, zero is a semisimple eigenvalue (if it is an eigenvalue) of the reversal $\lambda A_{0}+J+A_{1}$ of $P(\lambda)$ and hence the index of $P(\lambda)$ is at most one. This shows that $\mathcal{I}_{\text {hi }}$ is empty in that case.

Thus, assume that $j=k>1$. To show the inclusion $\overline{\mathcal{I}}_{\text {hi }} \subseteq \mathcal{K}$ suppose, as in the proof of (iii), that for some $P(\lambda) \in \mathcal{I}_{\text {hi }}$ we have ker $J \cap \operatorname{ker} A_{k} \cap \operatorname{ker} A_{k-1}=\{0\}$. If $\operatorname{ker}\left(A_{k}-J\right)=\{0\}$ then the matrix polynomial has no infinite eigenvalues and hence is of index zero. Hence, we may assume that $A_{k}-J$ has a nontrivial kernel, which by Proposition 9 applied with $\ell=0$ to $J$ and $X_{0}=A_{k}$ implies that there exists an orthogonal congruence transformation $U$ so that

$$
U^{\top} A_{k} U=\left[\begin{array}{cc}
\widetilde{A}_{k} & 0 \\
0 & 0
\end{array}\right], U^{\top} J U=\left[\begin{array}{cc}
J_{11} & 0 \\
0 & 0
\end{array}\right], \quad \text { and } \quad U^{\top} A_{k-1} U=\left[\begin{array}{cc}
A_{11} & A_{12} \\
A_{12}^{\top} & A_{22}
\end{array}\right],
$$

where $\widetilde{A}_{k}-J_{11} \in \mathbb{R}^{n-r, n-r}(r>0)$ is invertible and all three matrices are partitioned conformably. In fact, replacing $P(\lambda)$ by $U^{\top} P(\lambda) U$ if necessary, we may assume $U=I_{n}$ in what follows. Since ker $J \cap \operatorname{ker} A_{k} \cap \operatorname{ker} A_{k-1}=\{0\}$ and since $A_{k-1}$ is positive semidefinite, it follows that $A_{22}$ is invertible. The companion matrix pencil (25) of $P(\lambda)$ takes the form

$$
\lambda\left[\begin{array}{cc|c}
\widetilde{A}_{k}-J_{11} & \\
& 0 & \\
\hline & I_{(k-1) n}
\end{array}\right]+\left[\begin{array}{cc|c}
A_{11} & A_{12} & * \\
A_{12}^{\top} & A_{22} & * \\
\hline * & * & *
\end{array}\right]
$$

and from Lemma 3 we infer that the matrix polynomial $P(\lambda)$ is of index one.

For the converse $\mathcal{K} \subseteq \overline{\mathcal{I}}_{\text {hi }}$ let $P(\lambda) \in \mathcal{K}$. Then we have the following decomposition

$$
\begin{gathered}
U^{\top} A_{k} U=\left[\begin{array}{cc}
\widetilde{A}_{k} & 0 \\
0 & 0
\end{array}\right], U^{\top} A_{k-1} U=\left[\begin{array}{cc}
\widetilde{A}_{k-1} & 0 \\
0 & 0
\end{array}\right], U^{\top} J U=\left[\begin{array}{cc}
J_{11} & 0 \\
0 & 0
\end{array}\right], \\
\text { and } U^{\top} A_{k-2} U=\left[\begin{array}{cc}
A_{11} & A_{12} \\
A_{12}^{\top} & A_{22}
\end{array}\right], U^{\top} J U=\left[\begin{array}{cc}
J_{11} & 0 \\
0 & 0
\end{array}\right],
\end{gathered}
$$

with $A_{k}, A_{k-1}, A_{11}, J_{11} \in \mathbb{R}^{n-1, n-1}$ not necessarily invertible. Replacing $\widetilde{A}_{k-2}$ with $\widetilde{A}_{k-2}+$ $\varepsilon I_{n-1}$, then for sufficiently small $\varepsilon$ we we get a family of regular pencils $P_{\varepsilon}(\lambda)$ of index at least two and such that $\mathcal{I}_{\text {hi }} \ni P_{\varepsilon}(\lambda) \rightarrow P(\lambda) \in \overline{\mathcal{I}}_{\text {hi }}$.

(iv) is an immediate consequence of (iii). 
Remark 18 At first, it may come as a surprise that a matrix polynomial $P(\lambda)$ as in Theorem 16 is already singular if $P(1)$ is singular which means that 1 cannot be an eigenvalue of a regular $P(\lambda) \in \mathcal{P}_{k, j}^{n}$. More generally, if $\alpha>0$ then replacing $\lambda$ with $\frac{\lambda}{\alpha}$ and $A_{i}$ with $\alpha^{i} A_{i}$ shows that if $P(\alpha)$ is singular, then $P(\lambda)$ is already a singular matrix polynomial. This generalizes in a nontrivial way the observation that any scalar polynomial with nonnegative coefficients cannot have real zeros that are positive unless it is the zero polynomial.

Remark 19 The reason for not investigating the structured distance to instability for $P(\lambda)$ in Theorem 16 is the fact that in contrast to Theorem [6(iii) the distances to higher index and instability need not coincide for matrix polynomials of degree larger than one. We will return to the distance to instability for quadratic polynomials in Section 6.2, because that task is still accessible by the common kernel methods framework. This is due to a nontrivial result, Theorem 27 below, which states that the only spectral points that may cause instability are zero and infinity. However, already for degree three the reason for instability may be different, since a polynomial in $\mathcal{P}_{3, \ell}^{n}$ might have eigenvalues in the right half plane. For example, the scalar polynomial $p(\lambda)=\lambda^{3}+1 \in \mathcal{P}_{3, \ell}^{1}$ (thus $J=X_{1}=X_{2}=0, X_{0}=X_{3}=1$ ) has two roots in the right half plane.

\section{Distance problems for first order dHDAE systems}

In this section we will revisit the distance problems for first order dHDAE systems formulated in Section 3. We will first present the missing proofs which are now easy consequences of the extended results from the previous section. Then, we will present two examples that show that the structured distances for dHDAE systems may differ considerably from the corresponding ones under arbitrary perturbations.

\subsection{Proofs of and comments on the main results in Section 3}

Proof of Theorem 5. (ii) It follows from Theorem [16(ii) $(k=1, j=0)$ that $P(\lambda)$ is singular if and only if the kernels of $E, J$ and $R$ have a nontrivial intersection. Applying Theorem 9 $(\ell=1)$ we get the desired transformation $U$.

(iii) By Theorem 2 the index is at most two. Then it follows from Theorem 16)(iii) $(k=1$, $j=0$ ) that $P(\lambda)$ is in the closure of regular $\mathrm{dH}$ pencils of index 2 if and only if the kernels of $E$ and $R$ have a nontrivial intersection.

Proof of Theorem 6. (ii) The proof is obtained from Theorem 13 with $k=1, X_{0}=E$ and $X_{1}=R$ and Theorem [16, and using $J^{\top} J=-J^{2}$.

(iii) First, it immediately follows from Theorem 2 that $P(\lambda)$ is stable if and only if it is regular and of index one. The proof is then obtained from Theorem 13 with $k=1, X_{0}=E$ and $X_{1}=R$ and using $J^{\top} J=-J^{2}$. By Theorem 2 any pencil $\lambda E-(J-R)$ with $E, R \geq 0$ and $J^{\top}=-J$, associated with a $\mathrm{dH}$ system, is of index at most 2 .

We have the following immediate corollary of Theorems 5 and 6 .

Corollary 20 For $J=-J^{\top}, E, R \geq 0$ one has the following estimate

$2 \cdot \lambda_{\min }\left(-J^{2}\right)+d_{\mathrm{hi}}^{\mathcal{L}}(\lambda E-(J-R))^{2} \leq d_{\text {sing }}^{\mathcal{L}}(\lambda E-(J-R))^{2} \leq 2\|J\|^{2}+d_{\text {hi }}^{\mathcal{L}}(\lambda E-(J-R))^{2}$.

In particular, the set of singular $d H$ pencils in $\mathcal{P}_{1,0}^{n}$ is contained in the closure in $\mathcal{P}_{1,0}^{n}$ of the set of index two regular $d H$ pencils. 
Remark 21 Consider the reversed pencil $-\lambda(J-R)+E$ with $J=-J^{\top}$ and $E, R \geq 0$. Statement (iii) of Theorem [16] shows that its structured distance to higher index $d_{\mathrm{hi}}^{\mathcal{L}}(-\lambda(J-$ $R)+E$ ) equals its distance to singularity $d_{\text {sing }}^{\mathcal{L}}(-\lambda(J-R)+E)$. This is in line with the fact that $\infty$ can only be a semisimple eigenvalue of $-\lambda(J-R)+E$ as zero is a semisimple eigenvalue of $\lambda E-(J-R)$, cf. Theorem 5. Then, in summary, we have

$$
\begin{aligned}
d_{\mathrm{ker}}^{\mathcal{P}}(J, E, R) & =d_{\mathrm{hi}}^{\mathcal{P}}(-\lambda(J-R)+E) \\
& =d_{\mathrm{sing}}^{\mathcal{P}}(-\lambda(J-R)+E) \\
& =d_{\mathrm{sing}}^{\mathcal{P}}(\lambda E-(J-R)) \\
& =d_{\operatorname{sing}}^{\mathcal{P}}(\lambda R-(J-E)) .
\end{aligned}
$$

Since our main focus is on distance problems in this paper, it was necessary to characterize the closure of the set of $\mathrm{dH}$ pencils of index two in Theorem 5 (iii). Our next result gives a characterization of the set of regular $\mathrm{dH}$ pencils of index two.

Proposition 22 A pencil $L(\lambda)=\lambda E-(J-R)$ with $E, R \geq 0, J=-J^{\top}$ is regular of index two if and only if there exists an orthogonal matrix $U$ such that

$U^{\top} E U=\left[\begin{array}{ccc}E_{11} & 0 & 0 \\ 0 & 0 & 0 \\ 0 & 0 & 0\end{array}\right], \quad U^{\top} J U=\left[\begin{array}{ccc}J_{11} & J_{12} & J_{13} \\ -J_{12} & J_{22} & 0 \\ -J_{13}^{\top} & 0 & 0\end{array}\right], \quad U^{\top} R U=\left[\begin{array}{ccc}R_{11} & R_{12} & 0 \\ R_{12}^{\top} & R_{22} & 0 \\ 0 & 0 & 0\end{array}\right]$,

where $n=p+q+r, p, r>0, E_{11} \in \mathbb{R}^{p, p}$ is invertible, $J_{22}-R_{22} \in \mathbb{R}^{q, q}$ is invertible, and $J_{13} \in \mathbb{R}^{p, r}$ has full column rank.

Proof. Suppose that the decomposition (41) holds. As $J_{22}-R_{22}$ is invertible and $J_{13}$ has full column rank, we have that $-J+E+R$ is invertible. Hence, by Theorem 5(ii) and Theorem 16(1) the pencil is regular. Therefore, by Lemma 3 it is of index two. To prove the converse implication first we find an orthogonal transformation $U_{1}$ such that

$$
U_{1}^{\top} E U_{1}=\left[\begin{array}{cc}
E_{11} & 0 \\
0 & 0
\end{array}\right], \quad U_{1}^{\top} J U_{1}=\left[\begin{array}{cc}
J_{11} & J^{\prime} \\
-J^{\prime \top} & \widetilde{J}
\end{array}\right], \quad U_{1}^{\top} R U_{1}=\left[\begin{array}{cc}
R_{11} & R^{\prime} \\
R^{\prime \top} & \widetilde{R}
\end{array}\right],
$$

with $E_{11} \in \mathbb{R}^{p, p}$ invertible. By Lemma 3 the matrix $\widetilde{J}-\widetilde{R}$ is singular. Applying Theorem 9 to $\widetilde{J}$ and $\widetilde{R}$ we get an orthogonal transformation and a splitting of the last $n-p$ rows and columns, which combined with (42) gives

$$
U^{\top} E U=\left[\begin{array}{ccc}
E_{11} & 0 & 0 \\
0 & 0 & 0 \\
0 & 0 & 0
\end{array}\right], U^{\top} J U=\left[\begin{array}{ccc}
J_{11} & J_{12} & J_{13} \\
-J_{12}{ }^{\top} & J_{22} & 0 \\
-J_{13}^{\top} & 0 & 0
\end{array}\right], U^{\top} R U=\left[\begin{array}{ccc}
R_{11} & R_{12} & R_{13} \\
R_{12}^{\top} & R_{22} & 0 \\
R_{13}^{\top} & 0 & 0
\end{array}\right],
$$

with some orthogonal $U$. As $R$ is positive semidefinite we have $R_{13}=0$. But then $J_{13}$ needs to have full column rank, otherwise the pencil would be singular.

\subsection{Structured vs. unstructured distances}

In this subsection we compare the structured and the unstructured distances. First note that the statements of Theorem 5 are not true without the structure assumptions on $E$ and $R$. While it is obvious that (ii) cannot hold for arbitrary pencils, we need a simple example to disprove an unstructured analogue of (iii). 
Example 23 Let $n=2, E=J=0, R=I_{2}$. Then setting $\widetilde{J}=J, \widetilde{R}=R$ and

$$
\widetilde{E}=\left[\begin{array}{ll}
0 & \varepsilon \\
0 & 0
\end{array}\right]
$$

we see that $\lambda \widetilde{E}-(\widetilde{J}-\widetilde{R})$ is regular and of index two (but not dissipative Hamiltonian). Letting $\varepsilon \rightarrow 0$, we find that $\lambda E-(J-R)$ is in the closure of regular pencils of index two although $\operatorname{ker} E \cap \operatorname{ker} R=\{0\}$.

To analyze the distances in Theorem [6, recall that in [7] the (unstructured) distance to singularity was defined as

$$
d_{\text {sing }}(\lambda E-A):=\inf \left\{\left\|\left[\Delta_{E}, \Delta_{A}\right]\right\|_{F} \mid \lambda\left(E+\Delta_{E}\right)+A+\Delta_{A} \text { is singular }\right\} .
$$

\section{Example 24 Let}

$$
E=\left[\begin{array}{ll}
0 & 0 \\
0 & 1
\end{array}\right], \quad J=\left[\begin{array}{cc}
0 & -0.5 \\
0.5 & 0
\end{array}\right], \quad R=\left[\begin{array}{ll}
0.18 & 0.42 \\
0.42 & 1.03
\end{array}\right] \geq 0 .
$$

Then (rounding the numerical results to four digits) we have

$$
\lambda_{\min }\left(-J^{2}+E^{2}+R^{2}\right)=0.5819, \quad \sigma_{\min }\left(\left[\begin{array}{c}
A \\
E
\end{array}\right]\right)=0.1908, \quad \sigma_{\min }\left(\left[\begin{array}{ll}
A & E
\end{array}\right]\right)=0.6056,
$$

where $\sigma_{\min }$ stands for the smallest singular value. The first equality implies, by Theorem 6(ii), that $\left.d_{\text {sing }}^{\mathcal{L}}(\lambda E-(J-R))\right) \geq 0.5819$, while the second and third equality imply, together with Corollary 3 of [7], that $d_{\text {sing }}(\lambda E-A)=0.1908$.

The next example shows mainly the same behaviour, though we refine the constraints. Namely, we show that if we change the constraint in the definition of (6) to $E+\Delta_{E}, R+$ $\Delta_{R}$ being symmetric (instead of being positive definite) then we get an essentially different distance.

Example 25 Consider a dissipative Hamiltonian system (3) with coefficients

$$
E=\left[\begin{array}{lllll}
1 & & & & \\
& 1 & & & \\
& & 1 & & \\
& & & 0 & \\
& & & & 0
\end{array}\right], \quad J=\left[\begin{array}{ccccc}
0 & 0 & 0 & 0 & -1 \\
0 & 0 & 0 & 1 & 1 \\
0 & 0 & 0 & -1 & -1 \\
0 & -1 & 1 & 0 & \varepsilon \\
1 & -1 & 1 & -\varepsilon & 0
\end{array}\right], \quad R=\left[\begin{array}{ccccc}
\alpha & 0 & 0 & 0 & 1 \\
0 & \alpha & 0 & 1 & 1 \\
0 & 0 & \alpha & 1 & 1 \\
0 & 1 & 1 & \varepsilon & 0 \\
1 & 1 & 1 & 0 & \varepsilon
\end{array}\right],
$$

where $\varepsilon>0$ and

$$
\alpha=\varepsilon^{-1} \cdot\left\|\left[\begin{array}{ll}
0 & 1 \\
1 & 1 \\
1 & 1
\end{array}\right]\right\|_{F}^{2}+1 .
$$

By Theorem 1.1 of [12] such choice of $\alpha$ makes $R>0$. Consider now the perturbation

$$
\Delta_{E}=0, \quad \Delta_{J}=\left[\begin{array}{lll}
0_{3 \times 3} & & \\
& & -\varepsilon
\end{array}\right], \quad \Delta_{R}=\left[\begin{array}{lll}
0_{3 \times 3} & & \\
& -\varepsilon & \\
& & -\varepsilon
\end{array}\right] .
$$


Clearly $\Delta_{J}=-\Delta_{J}^{\top}$ and $\Delta_{R}=\Delta_{R}^{\top}$, and $\Delta_{E}=\Delta_{E}^{\top}$. The pencil $\lambda E-\widehat{A}$ with

$$
\widehat{A}:=J+\Delta_{J}-\left(R+\Delta_{R}\right)=\left[\begin{array}{ccccc}
-\alpha & 0 & 0 & 0 & -2 \\
0 & -\alpha & 0 & 0 & 0 \\
0 & 0 & -\alpha & -2 & -2 \\
0 & -2 & 0 & 0 & 0 \\
0 & -2 & 0 & 0 & 0
\end{array}\right]
$$

is now singular, because one easily checks that $\operatorname{det}(\lambda E-\widehat{A}) \equiv 0$. In this way, we have constructed a perturbation with

$$
\left\|\left[\Delta_{J}, \Delta_{R}, \Delta_{E}\right]\right\|_{F}=2 \varepsilon
$$

such that the perturbed pencil is singular, but the perturbation is not structure-preserving, because the matrix $R+\Delta_{R}$ is now indefinite. Observe also that, unlike in Theorem (5)(i), $E$ and $\widehat{A}$ do not have a common right or left kernel. This means that in the Kronecker canonical form there are left and right minimal indices of size at least one.

On the other hand, we have

$$
J^{\top} J+E^{2}+R^{2}=\left[\begin{array}{ccccc}
3+\alpha^{2} & 0 & 2 & -\varepsilon & \alpha+\varepsilon \\
0 & 5+\alpha^{2} & 0 & \alpha+2 \varepsilon & \alpha \\
2 & 0 & 5+\alpha^{2} & \alpha & \alpha+2 \varepsilon \\
-\varepsilon & \alpha+2 \varepsilon & \alpha & 4+2 \varepsilon^{2} & 4 \\
\alpha+\varepsilon & \alpha & \alpha+2 \varepsilon & 4 & 6+2 \varepsilon^{2}
\end{array}\right]
$$

and a simple MATLAB calculation shows that we have $\lambda_{\min }\left(J^{\top} J+E^{2}+R^{2}\right)^{1 / 2} \geq 0.81$ for $\varepsilon \in$ $\left(10^{-1}, 10^{-6}\right)$. Hence, the smallest perturbation $\Delta_{E}, \Delta_{J}, \Delta_{R}$ that makes the pencil singular, while keeping $R+\Delta_{R} \geq 0, E+\Delta_{E} \geq 0$, and $J+\Delta_{J}$ skew-symmetric, satisfies

$$
\left\|\left[\Delta_{J}, \Delta_{R}, \Delta_{E}\right]\right\|_{F} \geq 0.81
$$

for $\varepsilon \in\left(10^{-1}, 10^{-6}\right)$ by Theorem 6(1).

Note that the pencil $\lambda E-(J-R)$ from Example23 also shows that Theorem6(iii) does not hold for unstructured perturbations. Indeed, that pencil is in the closure of regular pencils of index 2 , but $d_{\mathrm{hi}}^{\mathcal{P}}(\lambda E-(J-R)) \geq \lambda_{\min }\left(E^{2}+R^{2}\right)=1$.

As last example we consider the analysis of distance problems in circuit simulation.

Example 26 A simple RLC network, see, e.g., [3, 9, 13, 14, can be modeled by a dHDAE system of the form

$$
\underbrace{\left[\begin{array}{ccc}
G_{c} C G_{c}^{\top} & 0 & 0 \\
0 & L & 0 \\
0 & 0 & 0
\end{array}\right]}_{=: E}\left[\begin{array}{c}
\dot{V} \\
\dot{I}_{l} \\
\dot{I}_{v}
\end{array}\right]=\underbrace{\left[\begin{array}{ccc}
-G_{r} R_{r}^{-1} G_{r}^{\top} & -G_{l} & -G_{v} \\
G_{l}^{\top} & 0 & 0 \\
G_{v}^{\top} & 0 & 0
\end{array}\right]}_{=: J-R}\left[\begin{array}{c}
V \\
I_{l} \\
I_{v}
\end{array}\right],
$$

where $L>0, C>0, R_{r}>0$ are real symmetric matrices describing inductances, capacitances, and resistances, respectively. The subscripts $r, c, l$, and $v$ refer to the resistors, capacitors, inductors, and voltage sources, while $V, I$ denote voltage and current, respectively. The matrices $G_{c}, G_{l}, G_{r}, G_{v}$ encode the network topology, see [13] for details. Here, $J$ and $-R$ 
are defined to be the skew-symmetric and symmetric parts, respectively, of the matrix on the right hand side of (44). We see that $E, R \geq 0$ and $J=-J^{T}$.

It was shown in [13, Theorem 1] that the pencil $\lambda E-(J-R)$ is regular if and only if $G_{v}$ has full column rank and

$$
G_{1}:=\left[\begin{array}{llll}
G_{c} & G_{r} & G_{l} & G_{v}
\end{array}\right]
$$

has full row rank. Note that this equivalence is now a simple corollary of Proposition 6(1i). Indeed, $\lambda E-(J-R)$ is singular if and only if the kernels of the three matrices

$$
E=\left[\begin{array}{ccc}
G_{c} C G_{c}^{\top} & 0 & 0 \\
0 & L & 0 \\
0 & 0 & 0
\end{array}\right], \quad J=\left[\begin{array}{ccc}
0 & -G_{l} & -G_{v} \\
G_{l}^{\top} & 0 & 0 \\
G_{v}^{\top} & 0 & 0
\end{array}\right], \quad \text { and } \quad R=\left[\begin{array}{ccc}
G_{r} R_{r}^{-1} G_{r}^{\top} & 0 & 0 \\
0 & 0 & 0 \\
0 & 0 & 0
\end{array}\right]
$$

have a nontrivial intersection. Having in mind that $C, L$, and $R_{r}^{-1}$ are positive definite matrices, we immediately obtain that $x=\left[\begin{array}{lll}x_{1}^{\top} & x_{2}^{\top} & x_{3}^{\top}\end{array}\right]^{\top} \in \operatorname{ker} E \cap \operatorname{ker} J \cap \operatorname{ker} R$ if and only if $G_{c}^{\top} x_{1}=0, x_{2}=0$ as well as $G_{l}^{\top} x_{1}=0, G_{v}^{\top} x_{1}=0, G_{v} x_{3}=0$, and $G_{r}^{\top} x_{1}=0$ which in turn is equivalent to

$$
x_{1}^{\top} G_{1}=0, \quad x_{2}=0, \quad \text { and } \quad G_{v} x_{3}=0 .
$$

Thus, we see that $\lambda E-(J-R)$ is singular if and only if either $G_{1}$ does not have full row rank or $G_{v}$ does not have full column rank.

All this shows that regularity of the pencil $\lambda E-(J-R)$ depends only on the network topology, cf. Remark 1 in [13. As one can expect, the distance to singularity depends also on the values of matrices $L, C, R_{r}$. Observing that the matrix $-J^{2}+R^{2}+E^{2}$ has the form

$$
-J^{2}+R^{2}+E^{2}=\left[\begin{array}{ccc}
\left(G_{c} C G_{c}^{\top}\right)^{2}+\left(G_{r} R_{r}^{-1} G_{r}^{\top}\right)^{2}+G_{l} G_{l}^{\top}+G_{v} G_{v}^{\top} & 0 & 0 \\
0 & L^{2}+G_{l}^{\top} G_{l} & G_{l}^{\top} G_{v} \\
0 & G_{v}^{\top} G_{l} & G_{v}^{\top} G_{v}
\end{array}\right],
$$

we obtain by (11) that the structured distance to singularity is bounded by

$$
\lambda_{\min }\left(-J^{2}+R^{2}+E^{2}\right)^{1 / 2} \leq d_{\text {sing }}^{\mathcal{L}}(\lambda E-(J-R)) \leq 2 \cdot \lambda_{\min }\left(-J^{2}+R^{2}+E^{2}\right)^{1 / 2},
$$

where $\lambda_{\min }\left(-J^{2}+R^{2}+E^{2}\right)$ is the minimum of the two values

$$
\lambda_{\min }\left(\left(G_{c} C G_{c}^{\top}\right)^{2}+\left(G_{r} R_{r}^{-1} G_{r}^{\top}\right)^{2}+G_{l} G_{l}^{\top}+G_{v} G_{v}^{\top}\right) \quad \text { and } \quad \lambda_{\min }\left(\left[\begin{array}{cc}
L^{2}+G_{l}^{\top} G_{l} & G_{l}^{\top} G_{v} \\
G_{v}^{\top} G_{l} & G_{v}^{\top} G_{v}
\end{array}\right]\right) .
$$

In this section we have characterized the distances to singularity, high index and instability for linear constant coefficient $\mathrm{dH}$ systems. In the next section we extend these results to quadratic matrix polynomials.

\section{Quadratic polynomials, linearization, and removing $Q$}

In this section, we will show how the main results of the previous sections can be applied to more general situations. We first study how the transformation (linearization) of structured matrix polynomials to $\mathrm{dH}$ pencils can be performed and then apply the distance results to quadratic matrix polynomials. Finally we discuss the more general $\mathrm{dH}$ pencils in (10) and show how the multiplier $Q$ can be removed. 


\subsection{Dissipative Hamiltonian linearisations}

Consider a second order system of the form

$$
M \ddot{x}-(G-D) \dot{x}+K x=0
$$

with $M, G, D, K \in \mathbb{R}^{n, n}$ satisfying $M, D, K \geq 0$ and $G=-G^{\top}$. A companion linearization (25) of (45) is given by

$L(\lambda)=\lambda\left[\begin{array}{cc}M & 0 \\ 0 & I\end{array}\right]-\left[\begin{array}{cc}D-G & K \\ -I & 0\end{array}\right]=\lambda\left[\begin{array}{cc}M & 0 \\ 0 & I\end{array}\right]-\left(\left[\begin{array}{cc}G & I \\ -I & 0\end{array}\right]-\left[\begin{array}{cc}D & 0 \\ 0 & 0\end{array}\right]\right)\left[\begin{array}{cc}I & 0 \\ 0 & K\end{array}\right]$.

It satisfies the hypothesis of Theorem 2 with

$$
E=\left[\begin{array}{cc}
M & 0 \\
0 & I
\end{array}\right], \quad J=\left[\begin{array}{cc}
G & I \\
-I & 0
\end{array}\right], \quad R=\left[\begin{array}{cc}
D & 0 \\
0 & 0
\end{array}\right], \quad Q=\left[\begin{array}{cc}
I & 0 \\
0 & K
\end{array}\right],
$$

and thus we immediately have the following result.

Theorem 27 Let $P(\lambda)=\lambda^{2} M-\lambda(G-D)+K$, where $M, D, K, G \in \mathbb{C}^{n, n}$ with $G^{H}=-G$ and $M, D, K \geq 0$. Then the following statements hold.

(i) All eigenvalues of $P(\lambda)$ are in the closed left half complex plane and all finite nonzero eigenvalues on the imaginary axis are semisimple.

(ii) The possible length of Jordan chains of $P(\lambda)$, associated with either the eigenvalue $\infty$ or the eigenvalue zero, is at most two.

(iii) All left and all right minimal indices of $P(\lambda)$ are zero (if there are any).

Proof. The proof of (i) and the statement in (ii) on the length of the Jordan chains associated with the eigenvalue $\infty$ follows immediately from Theorem 2 , while the remaining statement of (ii) then follows by applying the already proved part of (ii) to the reversal $\lambda^{2} K+\lambda(G-D)+M$ of $P(\lambda)$, which has the same structure. To see (iii), observe that by Theorem 2 the left minimal indices of $L(\lambda)$ are all zero and the right minimal indices of $L(\lambda)$ are at most one. By [10, Theorem 5.10] the left minimal indices of $P(\lambda)$ coincide with those of $L(\lambda)$, and if $\varepsilon_{1}, \ldots, \varepsilon_{k}$ are the right minimal indices of $P(\lambda)$, then $\varepsilon_{1}+1, \ldots, \varepsilon_{k}+1$ are the right minimal indices of $L(\lambda)$. This implies that all minimal indices of $P(\lambda)$ are zero (if there are any).

Remark 28 One may be tempted to use the results on $\mathrm{dH}$ pencils with $Q=I$ to prove Theorem 27. i.e., to apply Theorem 5 instead of Theorem 2 and also to extend the result to polynomials of higher degree. However, as the constant term of the companion form (25) contains a principal submatrix

$$
\left[\begin{array}{cc}
Y_{k} & Y_{k-1} \\
-I_{n} & 0
\end{array}\right]
$$

which has a positive semidefinite symmetric part only when $Y_{k-1}=I$, in general one needs to consider different linearizations, e.g., $L(\lambda)$ to be from one of the classical linearizations classes in [24, 25] or a so-called Fiedler linearization, see, e.g., [11. However, one still meets the following general obstacles. 
1) As we have seen in Remark 19, a polynomial from $\mathcal{P}_{k, j}^{n}$ may have spectrum in the right half plane if $k>2$. Hence, no linearization of such a polynomial satisfies the hypothesis of Theorem 2 .

2) The index of a polynomial from $\mathcal{P}_{k, j}^{n}$ may be larger than two if $k>2$; consider e.g. $P(\lambda)=\lambda^{3} X_{3}+\lambda^{2} X_{2}+\lambda X_{1}+\lambda X_{0}-J$ with $X_{3}=X_{2}=X_{1}=J=0$ and $X_{0}=1$. The companion form is

$$
\lambda\left[\begin{array}{lll}
0 & & \\
& 1 & \\
& & 1
\end{array}\right]+\left[\begin{array}{ccc}
0 & 0 & 1 \\
-1 & & \\
& -1 &
\end{array}\right],
$$

which corresponds to a block of size three at $\infty$, i.e., $P(\lambda)$ is of index 3 . Hence, none of its index preserving (strong) linearizations satisfies the hypothesis of Theorem 2 ,

3) Let $P(\lambda) \in \mathcal{P}_{k, j}^{n}$ be a singular matrix polynomial with left minimal indices $\eta_{1}, \ldots, \eta_{\ell}(\ell>0)$ and right minimal indices $\varepsilon_{1}, \ldots, \varepsilon_{\ell}$; note that the numbers of left and right minimal indices coincide, because the matrix polynomial is square. Take $L(\lambda)$ as any of the structured linearizations in [11, 24, 25]. Then, there exists a number $q \in\{0,1,2, \ldots, k-1\}$, known in advance for a particular linearization, such that $L(\lambda)$ has the left minimal indices $\eta_{1}+q, \ldots, \eta_{\ell}+q$ and right minimal indices $\varepsilon_{1}+k-1-q, \ldots, \varepsilon_{\ell}+k-1-q$, see [10, 11]. Thus, even for $k=2$, the linearization $L(\lambda)$ cannot satisfy the hypothesis of Theorem 5 and for $k>2$ it cannot satisfy the hypothesis of Theorem 2 .

Hence, if $k>2$, then a linearization $L(\lambda)$ of $P(\lambda)$ cannot be a $\mathrm{dH}$ pencil with arbitrary $Q$ and if $k=2$ it can only be a $\mathrm{dH}$ pencil with (necessarily nontrivial) $Q$. It remains an open question to derive additional conditions on the matrix coefficients for a polynomial $P(\lambda) \in \mathcal{P}_{k, j}^{n}$ in order to guarantee that its spectrum is in the closed left half plane such that all eigenvalues on the imaginary axis (possible excluding zero and infinity) are semisimple.

In the next subsection we study the case of quadratic matrix polynomials with $\mathrm{dH}$ structure.

\subsection{Quadratic matrix polynomials with $\mathrm{dH}$ structure}

In the case of quadratic matrix polynomials with $\mathrm{dH}$ structure, i.e., if $P(\lambda)=\lambda^{2} A_{2}+\lambda A_{1}+$ $A_{0} \in \mathcal{P}_{2,1}^{n}$, where $A_{2}, A_{0}, A_{1}+A_{1}^{\top} \geq 0$, the structured distances to singularity, to higher index, and to instability were defined in (26), (27), and (28), respectively. Recalling that for a matrix $Y \in \mathbb{R}^{n, n}$ and a vector $u \in \mathbb{R}^{n}$ with $\|u\|_{2}=1$ the perturbation matrix $\Delta_{Y}^{u}$ in (15) is defined as $\Delta_{Y}^{u}=-u u^{\top} Y-Y u u^{\top}+u u^{\top} Y u u^{\top}$, we obtain the following result.

Theorem 29 Let $P(\lambda)=\lambda^{2} M-\lambda(G-D)+K \in \mathcal{P}_{2,1}^{n}$, i.e., $M, D, K, G \in \mathbb{R}^{n, n}$ with $G^{\top}=-G$ and $M, D, K \geq 0$.

(i) The matrix polynomial $P(\lambda)$ is singular if and only if all four matrices $M, D, K, G$ have a common kernel. In particular, the structured distance to singularity $d_{\text {sing }}^{\mathcal{P}}(P(\lambda))$ is attained for a perturbation of the form $\Delta_{M}^{u}, \Delta_{D}^{u}, \Delta_{K}^{u}, \Delta_{G}^{u}$ for some $u \in \mathbb{R}^{n, n}$ with $\|u\|_{2}=1$ and satisfies

$$
d_{\text {sing }}^{\mathcal{P}}(P(\lambda))=d_{\text {ker }}^{\mathcal{S}}(G, M, D, K),
$$

where $d_{\mathrm{ker}}^{\mathcal{S}}(G, M, D, K)$ is given by Theorem 13. In particular, the structured distance to singularity is bounded by

$$
\sqrt{\lambda_{\min }\left(M^{2}+D^{2}+K^{2}-G^{2}\right)} \leq d_{\text {sing }}^{\mathcal{P}}(P(\lambda)) \leq \sqrt{2 \cdot \lambda_{\min }\left(M^{2}+D^{2}+K^{2}-G^{2}\right)} .
$$


(ii) The matrix polynomial $P(\lambda)$ is in the closure of polynomials in $\mathcal{P}_{2,1}^{n}$ that are regular and of index larger than one (and thus of index exactly two) if and only if $M$ and $D$ have a common kernel. In particular, the structured distance to higher index $d_{\mathrm{hi}}^{\mathcal{P}}(P(\lambda))$ is attained for a perturbation of the form $\Delta_{M}^{u}, \Delta_{D}^{u}$, for some $u \in \mathbb{R}^{n, n}$ with $\|u\|_{2}=1$ and satisfies

$$
d_{\mathrm{hi}}^{\mathcal{P}}(P(\lambda))=d_{\mathrm{ker}}^{\mathcal{S}}(0, M, D),
$$

where $d_{\mathrm{ker}}^{\mathcal{S}}(0, M, D)$ is given by Theorem [13, In particular, the structured distance to higher index is bounded by

$$
\sqrt{\lambda_{\min }\left(M^{2}+D^{2}\right)} \leq d_{\mathrm{hi}}^{\mathcal{P}}(P(\lambda)) \leq \sqrt{2 \cdot \lambda_{\min }\left(M^{2}+D^{2}\right)} .
$$

(iii) The matrix polynomial $P(\lambda)$ is in the closure of polynomials in $\mathcal{P}$ that are unstable if and only if $M$ and $D$ have a common kernel or $D$ and $K$ have a common kernel. In particular, the structured distance to instability $d_{\text {inst }}^{\mathcal{D}}(P(\lambda))$ is attained for a perturbation of the form $\Delta_{M}^{u}, \Delta_{D}^{u}, \Delta_{K}^{u}$ with $\Delta_{M}^{u}=0$ or $\Delta_{K}^{u}=0$ for some $u \in \mathbb{R}^{n, n}$ with $\|u\|_{2}=1$ and satisfies

$$
d_{\text {inst }}^{\mathcal{P}}(P(\lambda))=\min \left\{d_{\text {ker }}^{\mathcal{S}}(0, M, D), d_{\text {ker }}^{\mathcal{S}}(0, D, K)\right\}
$$

where $d_{\mathrm{ker}}^{\mathcal{S}}(0, M, D)$ and $d_{\mathrm{ker}}^{\mathcal{S}}(0, D, K)$ are given by Theorem 13 , Moreover, the distance to instability is bounded by

$$
\sqrt{\alpha} \leq d_{\text {inst }}^{P}(P(\lambda)) \leq \sqrt{2 \cdot \alpha}
$$

where $\alpha:=\min \left\{\lambda_{\min }\left(M^{2}+D^{2}\right), \lambda_{\min }\left(D^{2}+K^{2}\right)\right\}$.

Proof. Parts (ii) and (iii) immediately follow from Theorem 16, For part (iii), we can apply Theorem 27, if the matrix polynomial $P(\lambda)$ is singular, regular of index two, or if it is regular and has a Jordan block of size two at $\lambda=0$, which is equivalent to the reversal $\lambda^{2} K+\lambda(D-G)+M$ of $P(\lambda)$ having index two. In all cases the statement follows immediately from (iii) applied to $P(\lambda)$ and its reversal, which has the same structure.

\subsection{Removing the coefficient $Q$ in $\mathrm{dH}$ systems}

Due to the multiplicative structure, in the model representation (11) the coefficient $Q$ will present difficulties for the perturbation analysis and it is an open question whether the definition of a $\mathrm{dH}$ system needs this term at all. In this section we will show that the factor $Q$ can be removed and the system (3) can be reduced to a system with $Q=I_{n}$.

Suppose first that $Q$ is invertible. Then the system (11) is equivalent to the system

$$
Q^{\top} E \dot{x}=Q^{\top}(J-R) Q x .
$$

Then setting $\tilde{Q}=I, \tilde{E}=Q^{\top} E, \tilde{J}=Q^{\top} J Q, \tilde{R}=Q^{\top} R Q$, we see that for the transformed system the constraints (2) are satisfied.

If $Q$ is not invertible then, using the singular value decomposition, there exist orthogonal matrices $U \in \mathbb{R}^{n, n}$ and $V \in \mathbb{R}^{n, n}$ such that

$$
U^{\top} Q V=\left[\begin{array}{cc}
Q_{11} & 0 \\
0 & 0
\end{array}\right], \quad U^{\top} E V=\left[\begin{array}{cc}
E_{11} & E_{12} \\
E_{21} & E_{22}
\end{array}\right], \quad U^{\top}(J-R) U=\left[\begin{array}{ll}
L_{11} & L_{12} \\
L_{21} & L_{22}
\end{array}\right]
$$


where in all three block matrices the $(1,1)$ block is square of $\operatorname{size} r=\operatorname{rank}(Q)$ and $Q_{11}$ is invertible. Since $Q^{\top} E=E^{\top} Q$, we get $Q_{1}^{\top} E_{11}=E_{11}^{\top} Q_{1}$ and $E_{12}=0$, and the transformed system is given by a reduced $\mathrm{dH}$ system

$$
\left[\begin{array}{cc}
E_{11} & 0 \\
E_{21} & E_{22}
\end{array}\right]\left[\begin{array}{l}
\dot{x}_{1} \\
\dot{x}_{2}
\end{array}\right]=\left[\begin{array}{cc}
L_{11} Q_{11} & 0 \\
L_{21} Q_{11} & 0
\end{array}\right]\left[\begin{array}{l}
x_{1} \\
x_{2}
\end{array}\right]
$$

where $x_{1}(t) \in \mathbb{R}^{k}, x_{2}(t) \in \mathbb{R}^{n-k}$.

If the pencil $\lambda E-(J-R) Q$ is regular then also the pencil $\lambda E-Q$ is regular and has index at most one, see [30]. Then $E_{22}$ is invertible, and therefore the second block-row of the system reads as

$$
\dot{x}_{2}=E_{22}^{-1}\left(-E_{21} \dot{x}_{1}+L_{21} Q_{1} x_{1}\right),
$$

$x_{1}$ does not depend on $x_{2}$ and the variable $x_{2}$ can be removed from the system. Then the reduced system

$$
E_{11} \dot{x}_{1}=L_{11} Q_{11} x_{1}
$$

satisfies the structured assumption (2) with $Q_{11}$ being invertible, so we can apply the previous procedure to obtain a system as in (49) for the reduced system.

The reduced system (51) may have a different Jordan structure at the eigenvalue zero than system (1). Indeed, $\mathrm{dH}$ pencils with singular $Q$ may have Jordan blocks of size two at the eigenvalue zero (see [30] for examples), while it follows from Theorem 2 that the eigenvalue zero is semisimple if $Q$ is invertible. A Jordan block of size 2 at the eigenvalue 0 would also mean that the system is unstable.

We highlight that the reduction procedure is not advisable if $E_{22}$ is ill-conditioned. Also, note that due to the fact that the procedure involves nonorthogonal transformations, the distance to singularity may change considerably during the process. It is an open problem to characterize the distance to singularity for a system in the form (11).

When the solution of the second order system (45), or, equivalently, the solution of the quadratic eigenvalue problem for $P(\lambda)=\lambda^{2} M-\lambda(G-D)+K$ is considered, then the classical approach is the linearization of the problem. As remarked in the proof of Theorem 27, a particular linearization of $P(\lambda)$ is of the form $\lambda E(J-R) Q$ with

$$
E=\left[\begin{array}{cc}
M & 0 \\
0 & I
\end{array}\right], \quad J=\left[\begin{array}{cc}
G & -I \\
I & 0
\end{array}\right], \quad R=\left[\begin{array}{cc}
D & 0 \\
0 & 0
\end{array}\right], \quad Q=\left[\begin{array}{cc}
I & 0 \\
0 & K
\end{array}\right]
$$

which corresponds to a system of the form (11). In this case we can remove the matrix $Q$ in a simpler way. Since $Q=Q^{\top}$ one case use $U=V$, and that $\operatorname{ker} Q=\{0\} \oplus \operatorname{ker} K$ to reduce the system to the form

$$
Q_{1}:=U^{\top} Q U=\left[\begin{array}{cc}
I & 0 \\
0 & K_{1}
\end{array}\right], \quad U^{\top} K U=\left[\begin{array}{cc}
K_{1} & 0 \\
0 & 0
\end{array}\right]
$$

with some symmetric positive $K_{1} \in \mathbb{R}^{k, k}$. As a result we get a so called trimmed linearization, see [8], i.e., a pencil $\lambda E_{1}-\left(J_{1}-R_{1}\right) \in \mathbb{R}^{n+k, n+k}$, where

$$
E_{1}=\left[\begin{array}{cc}
M & 0 \\
0 & K_{1}
\end{array}\right], \quad J_{1}=\left[\begin{array}{cc}
G & -K_{2}^{\top} \\
K_{2} & 0
\end{array}\right], \quad R_{1}=\left[\begin{array}{cc}
D & 0 \\
0 & 0
\end{array}\right]
$$


and $K_{2}=\left[\begin{array}{ll}K_{1} & 0\end{array}\right] \in \mathbb{R}^{k, n}$. Our aim is now to provide results that allow to compare the distances for the trimmed linearisation $\lambda E_{1}-\left(J_{1}-R_{1}\right)$ with the original distances obtained in Theorem 29, In the distances for the reduced system we use the matrices

$$
-J_{1}^{2}+E_{1}^{2}+R_{1}^{2}=\left[\begin{array}{cc}
M^{2}+G^{\top} G+K_{2}^{\top} K_{2}+D^{2} & -G K_{2}^{\top} \\
K_{2} G & K_{2} K_{2}^{\top}
\end{array}\right]
$$

and

$$
E_{1}^{2}+R_{1}^{2}=\left[\begin{array}{cc}
M^{2}+D^{2} & 0 \\
0 & K_{2} K_{2}^{\top}
\end{array}\right]
$$

Hence, $\lambda_{\min }\left(-J_{1}^{2}+E_{1}^{2}+R_{1}^{2}\right) \leq \lambda_{\min }\left(K_{2} K_{2}^{\top}\right)=\lambda_{\min }\left(K_{1}^{2}\right)$ and if $G=0$ the inequality becomes an equality. We get immediately the following result.

Proposition 30 For the reduced sytem (53) one has the following statements.

(i) The structured distance to singularity of the pencil $\lambda E_{1}-\left(J_{1}-R_{1}\right) \in \mathcal{L}$ satisfies

$$
d_{\text {sing }}^{\mathcal{L}}\left(\lambda E_{1}-\left(J_{1}-R_{1}\right)\right) \leq \sqrt{2 \cdot \lambda_{\min }\left(K_{1}^{2}\right)},
$$

while for $G=0$ we have

$$
\lambda_{\min }\left(K_{1}^{2}\right) \leq d_{\text {sing }}^{\mathcal{L}}\left(\lambda E_{1}-\left(J_{1}-R_{1}\right)\right) .
$$

(ii) The structured distance to higher index of the pencil $\lambda E_{1}-\left(J_{1}-R_{1}\right) \in \mathcal{L}$ satisfies

$$
\sqrt{\beta} \leq d_{\mathrm{hi}}^{\mathcal{L}}\left(\lambda E_{1}-\left(J_{1}-R_{1}\right)\right) \leq \sqrt{2 \cdot \beta},
$$

where $\beta=\min \left\{\lambda_{\min }\left(M^{2}+D^{2}\right), \lambda_{\min }\left(K_{1}^{2}\right)\right\}$.

\section{Conclusions}

Distance problems in linear differential-algebraic systems with dissipative Hamiltonian structure have been studied. These include the distance to the nearest singular problem, the distance to the nearest high index problem, and the distance to instability. The characterization of these distances are open problems for general linear differential-algebraic systems, while we have shown that for dissipative Hamiltonian systems and related matrix polynomials, explicit characterizations in terms of common null-spaces of several matrices exist.

\section{References}

[1] N. Aliyev, V. Mehrmann, and E. Mengi. Computation of stability radii for large-scale dissipative hamiltonian systems. Advances Comp. Math., To appear, 2020.

[2] C. Beattie, V. Mehrmann, and P. Van Dooren. Robust port-Hamiltonian representations of passive systems. Automatica, 100:182-186, 2019.

[3] C. Beattie, V. Mehrmann, H. Xu, and H. Zwart. Port-Hamiltonian descriptor systems. Math. Control, Signals, Sys., 30:17, 2018. https://doi.org/10.1007/s00498-018-0223-3. 
[4] T. Berger, H. Gernandt, C. Trunk, H. Winkler, and M. Wojtylak. A new bound for the distance to singularity of a regular matrix pencil. In Proc. Appl. Mathematics and Mechanics, volume 17.1, pages 863-864. Wiley Online Library, 2017.

[5] T. Berger, H. Gernandt, C. Trunk, H. Winkler, and M. Wojtylak. The gap distance to the set of singular matrix pencils. Linear Algebra Appl., 564:28-57, 2019.

[6] K. E. Brenan, S. L. Campbell, and L. R. Petzold. Numerical Solution of Initial-Value Problems in Differential Algebraic Equations. SIAM Publications, Philadelphia, PA, 2nd edition, 1996.

[7] R. Byers, C. He, and V. Mehrmann. Where is the nearest non-regular pencil. Linear Algebra Appl., 285:81-105, 1998.

[8] R. Byers, V. Mehrmann, and H. Xu. Trimmed linearizations for structured matrix polynomials. Linear Algebra Appl., 429:2373-2400, 2008.

[9] L. Dai. Singular Control Systems. Number 118 in Lecture Notes in Control and Information Sciences. Springer-Verlag, Berlin, 1989.

[10] F. De Terán, F. Dopico, and D. S. Mackey. Linearizations of singular matrix polynomials and the recovery of minimal indices. Electron. J. Linear Algebra, 18:371-402, 2009.

[11] F. De Terán, F. Dopico, and D. S. Mackey. Fiedler companion linearizations and the recovery of minimal indices. SIAM J. Matrix Anal. Appl., 31(4):2181-2204, 2010.

[12] C. Foias and A. E. Frazho. Positive definite block matrices. In The Commutant Lifting Approach to Interpolation Problems, pages 547-586. Springer, 1990.

[13] R. W. Freund. Structure-preserving model order reduction of rcl circuit equations. In Model Order Reduction: Theory, Research Aspects and Applications, pages 49-73. Springer, 2008.

[14] R. W. Freund. The SPRIM algorithm for structure-preserving order reduction of general rcl circuits. In Model reduction for circuit simulation, pages 25-52. Springer, 2011.

[15] F. R. Gantmacher. Theory of Matrices, volume 1. Chelsea, New York, 1959.

[16] N. Gillis, V. Mehrmann, and P. Sharma. Computing nearest stable matrix pairs. Numer. Lin. Alg. Appl., 25:e2153, 2018.

[17] N. Gillis and P. Sharma. On computing the distance to stability for matrices using linear dissipative hamiltonian systems. Automatica, 85:113-121, 2017.

[18] N. Gillis and P. Sharma. Finding the nearest positive-real system. SIAM J. Matrix Anal. Appl., 56(2):1022-1047, 2018.

[19] I. Gohberg, M. A. Kaashoek, and P. Lancaster. General theory of regular matrix polynomials and band Toeplitz operators. Integral Equations Operator Theory, 11(6):776-882, 1988. 
[20] N. Gräbner, V. Mehrmann, S. Quraishi, C. Schröder, and U. von Wagner. Numerical methods for parametric model reduction in the simulation of disc brake squeal. Z. Angew. Math. Mech., 96:1388-1405, 2016.

[21] N. Guglielmi, C. Lubich, and Volker Mehrmann. On the nearest singular matrix pencil. SIAM J. Matrix Anal. Appl., 38(3):776-806, 2017.

[22] B. Jacob and H. Zwart. Linear port-Hamiltonian systems on infinite-dimensional spaces. Operator Theory: Advances and Applications, 223. Birkhäuser/Springer Basel AG, Basel CH, 2012.

[23] P. Kunkel and V. Mehrmann. Differential-Algebraic Equations. Analysis and Numerical Solution. EMS Publishing House, Zürich, Switzerland, 2006.

[24] D. S. Mackey, N. Mackey, C. Mehl, and V. Mehrmann. Structured polynomial eigenvalue problems: Good vibrations from good linearizations. SIAM J. Matrix Anal. Appl., 28(4):1029-1051, 2006.

[25] D. S. Mackey, N. Mackey, C. Mehl, and V. Mehrmann. Vector spaces of linearizations for matrix polynomials. SIAM J. Matrix Anal. Appl., 28(4):971-1004, 2006.

[26] D.S. Mackey, N. Mackey, C. Mehl, and V. Mehrmann. Smith forms for palindromic matrix polynomials. Electron. J. Linear Algebra, 22:53-91, 2011.

[27] C. Mehl, V. Mehrmann, and P. Sharma. Stability radii for linear hamiltonian systems with dissipation under structure-preserving perturbations. SIAM Matrix Anal. Appl., 37:1625-1654, 2016.

[28] C. Mehl, V. Mehrmann, and P. Sharma. Stability radii for real linear Hamiltonian systems with perturbed dissipation. BIT, Numer. Math., 57:811-843, 2017.

[29] C. Mehl, V. Mehrmann, and M. Wojtylak. On the distance to singularity via low rank perturbations. Operators and Matrices, 9:733-772, 2015.

[30] C. Mehl, V. Mehrmann, and M. Wojtylak. Linear algebra properties of dissipative Hamiltonian descriptor systems. SIAM J. Matrix Anal. Appl., 39(3):1489-1519, 2018.

[31] V. Mehrmann and R. Morandin. Structure-preserving discretization for port-hamiltonian descriptor systems. In 58th IEEE Conference on Decision and Control (CDC), Nice, pages 6863-6868, 2019. https://arXiv:1903.10451.

[32] A. J. van der Schaft. Port-Hamiltonian differential-algebraic systems. In Surveys in Differential-Algebraic Equations I, pages 173-226. Springer-Verlag, 2013.

[33] A. J. van der Schaft and B. M. Maschke. Hamiltonian formulation of distributedparameter systems with boundary energy flow. J. Geom. Phys., 42:166-194, 2002.

[34] A. van der Schaft and B. Maschke. Generalized port-Hamiltonian dae systems. Systems $\&$ Control Letters, 121:31-37, 2018.

[35] A. J. van der Schaft and D. Jeltsema. Port-Hamiltonian systems theory: An introductory overview. Foundations and Trends in Systems and Control, 1(2-3):173-378, 2014. 